Aus dem Institut für Diagnostische und Interventionelle Neuroradiologie (Prof. Dr. med. M. Knauth) der Medizinischen Fakultät der Universität Göttingen

\title{
Retrospektive Evaluation retinaler Nervenfaserschichtdicke mit der cerebralen T2w- Läsionslast im MRT sowie dem Expanded Disability Status Scale (EDSS) bei pädiatrischen Patienten mit Multipler Sklerose
}

\author{
INAUGURAL-DISSERTATION \\ zur Erlangung des Doktorgrades \\ der Medizinischen Fakultät der \\ Georg-August-Universität zu Göttingen \\ vorgelegt von \\ Omar Al-Bourini \\ aus
}

Hannover

Göttingen 2017 
Dekan:

Referent/in

Ko-Referent/in:
Prof. Dr. rer. nat. H.K. Kroemer

Prof. Dr. med. M. Knauth

PD Dr. S. Dreha-Kulaczewski

Datum der mündlichen Prüfung: 12.06 .2018 
Hiermit erkläre ich, die Dissertation mit dem Titel "Retrospektive Evaluation retinaler Nervenfaserschichtdicke mit der cerebralen T2w-Läsionslast im MRT sowie dem Expanded Disability Status Scale (EDSS) bei pädiatrischen Patienten mit Multipler Sklerose" eigenständig angefertigt und keine anderen als die von mir angegebenen Quellen und Hilfsmittel verwendet zu haben.

Göttingen, den

(Omar Al-Bourini) 


\section{Inhaltsverzeichnis}

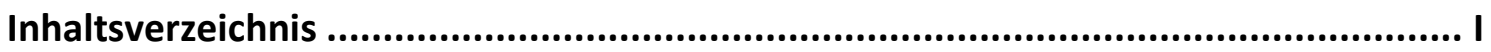

Abbildungsverzeichnis............................................................................................ III

Tabellenverzeichnis................................................................................................ III

Abkürzungsverzeichnis........................................................................................... IV

$1 \quad$ Einleitung .............................................................................................

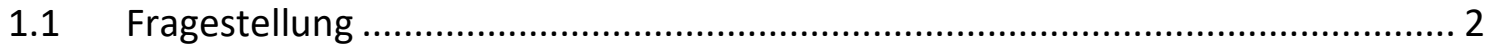

1.2 Multiple Sklerose und deren gesellschaftliche Bedeutung .................................. 2

1.3 Multiple Sklerose bei Kindern ...................................................................... 4

1.4 Optische Kohärenztomographie und Multiple Sklerose ……………………...... 7

1.5 Magnetresonanztomographie und Multiple Sklerose ………………………..... 9

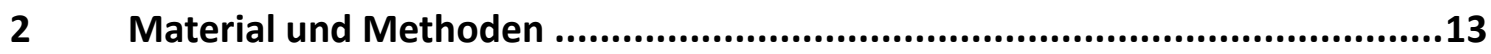

2.1 Studiendesign und Auswahlkriterien ............................................................... 13

2.2 Die Optische Kohärenztomographie (OCT) ..................................................... 14

2.3 Die Magnetresonanztomographie (MRT) und Aufnahmetechnik ...................... 14

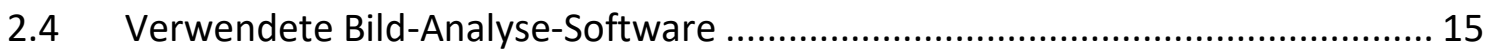

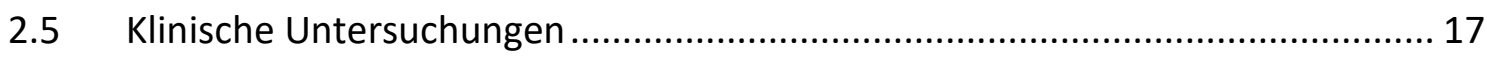

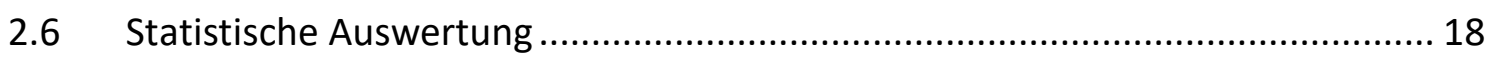

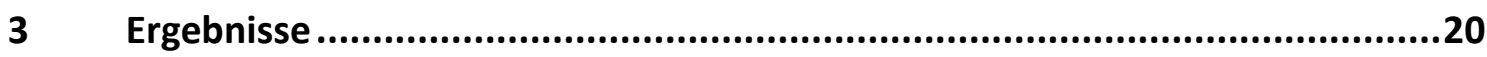

3.1 Epidemiologie und Aufbau der Kohorte .......................................................... 20

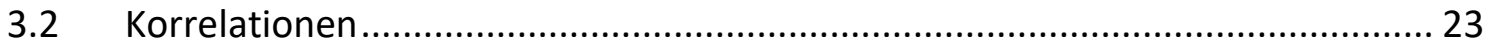

3.3 Ergebnisse der OCT-Untersuchungen .......................................................... 24

3.4 Ergebnisse hinsichtlich der MRT-Untersuchungen ........................................ 24

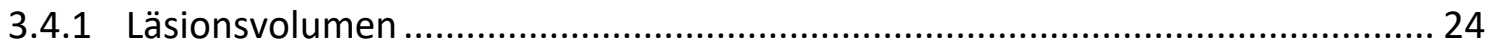

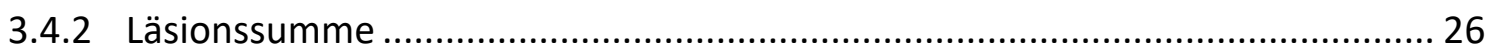

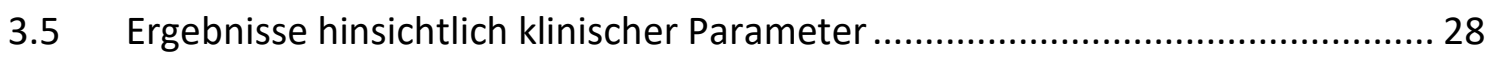

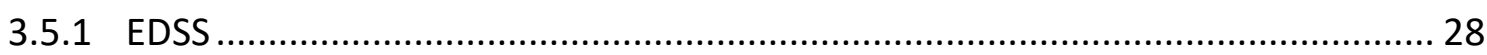

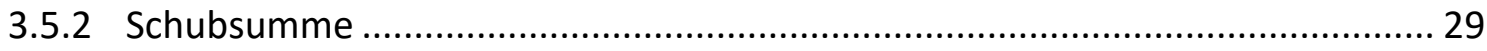

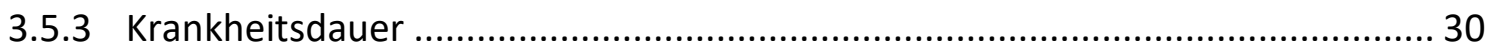

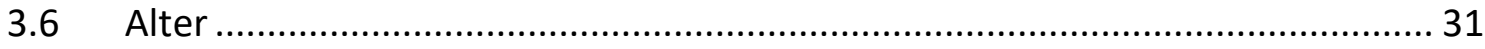

$4 \quad$ Diskussion ..................................................................................................3 


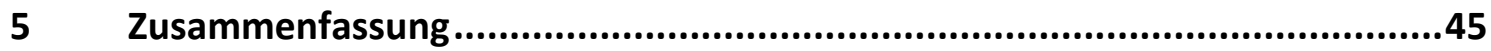

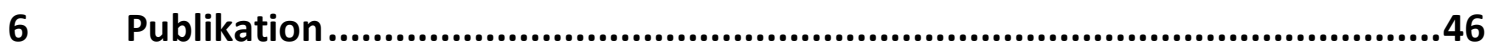

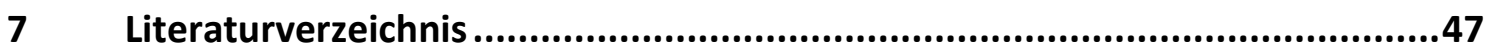

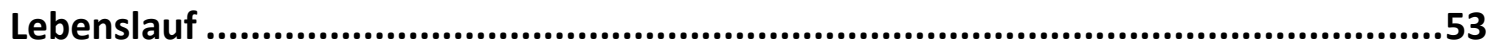




\section{Abbildungsverzeichnis}

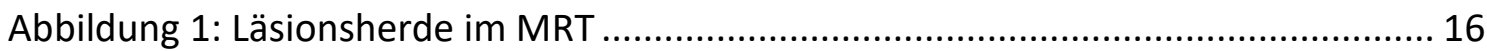

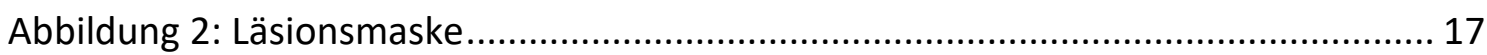

Abbildung 3: Verteilung der RNFLT $(\mu \mathrm{m})$ nach nNNO und NNO Augen .......................... 20

Abbildung 4: RNFLT OS $(\mu \mathrm{m})$ vs. RNFLT OD $(\mu \mathrm{m})$; (nNNO bilateral; $\mathrm{n}=29$ Patienten) .... 21

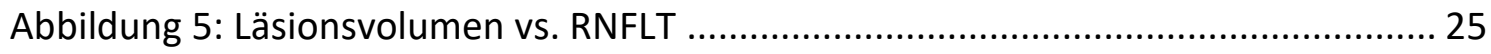

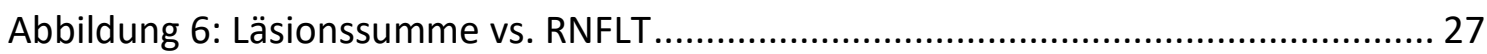

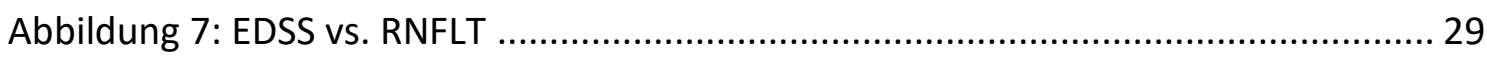

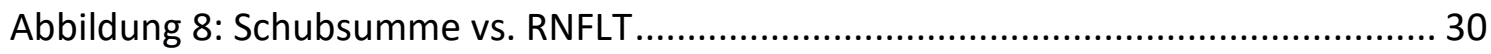

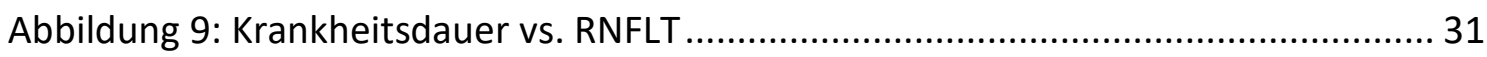

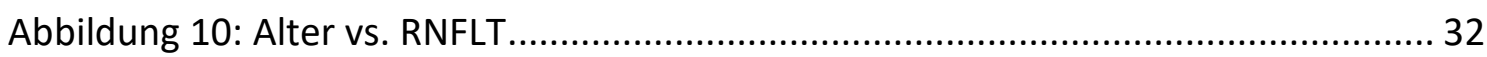

\section{Tabellenverzeichnis}

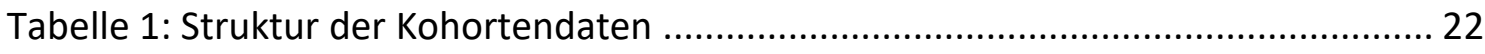

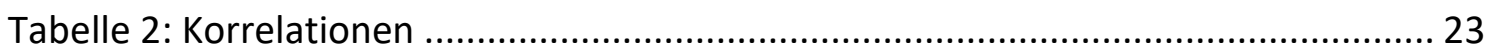




\section{Abkürzungsverzeichnis}

DR: $\quad$ antigen D-related

EDSS: Expanded Disability Status Scale

F: $\quad$ Female

FLAIR: $\quad$ Fluid-Attenuated Inversion Recovery

HLA: Humanes Leukozyten-Antigen

$\mathrm{M}$ : $\quad$ Male

$\mathrm{mm}$ : Millimeter

$\mathrm{mm}^{3}$ : Kubikmillimeter

MR: Magnetresonanz

MRT: Magnetresonanztomographie

MS: $\quad$ Multiple Sklerose

ms: Millisekunden

MW: Mittelwert

$\mu \mathrm{m}$ : Mikrometer

NAWM: Normal Appearing White Matter

nNNO: non-Neuritis nervi optici

NNO: Neuritis nervi optici

OCT: Optische Kohärenztomographie

OD: $\quad$ Oculus dexter

OS: Oculus sinister

p: probability

RNFLT: Retinal Nerve Fiber Layer Thickness

ROI: Region of Interest

SD: $\quad$ Standard Deviation (Standardabweichung)

SE: $\quad$ Standard Error (Standardfehler)

T2w: T2-Wichtung

TR: Repetition Time

UMG: Universitätsmedizin Göttingen

vs.: versus

ZNS: Zentrales Nervensystem 


\section{$1 \quad$ Einleitung}

Die Multiple Sklerose (MS) ist die häufigste zu chronischen Behinderungen führende entzündliche Erkrankung des Nervensystems. Obwohl noch kein Auslöser bekannt ist, geht man von einer multifaktoriellen autoimmunen Genese aus. Sie betrifft vor allem das junge Erwachsenenalter, kann jedoch bereits im frühen Kindesalter auftreten.

Wie nachfolgend dargelegt, richtet sich die Standarddiagnostik der Multiplen Sklerose bei Kindern und Erwachsenen nach den sogenannten McDonald-Kriterien (McDonald et al. 2001). Diese stellen ein Klassifikationssystem dar, welches u. a. auf neuroradiologischen Verfahren basiert, wie beispielsweise der Magnetresonanztomograhie (MRT) (McDonald et al. 2001). Die Korrelation der Diagnostik mittels MRT mit klinischen Befunden wurde durch Studien, die das sogenannte Klinisch-Radiologische Paradoxon thematisieren (Barkhof 2002), infrage gestellt. Dieses beschreibt die diagnostische Lücke zwischen der radiologischen Nachweisbarkeit MS-typischer Veränderungen, wie beispielsweise dem Auftreten von Läsionsherden, und dem hiervon oftmals dissoziierten Beginn krankheitstypischer Symptome und Ausfallerscheinungen. Eine sich aufdrängende Frage, bei deren Beantwortung diese Dissertation unterstützend mitwirken soll, ist die nach der eventuellen Auflösbarkeit dieses Klinisch-Radiologischen Paradoxons unter Zuhilfenahme von diagnostischen Hilfsmitteln wie der Optischen Kohärenztomographie (OCT). Die OCT könnte im Vergleich zur MRT, insbesondere unter Berücksichtigung des häufig gleichzeitigen Auftretens von Läsionsherden in der Sehbahn und der Reduktion der retinalen Nervenfaserschichtdicke (Sinnecker et al. 2015), eine zeit- und kosteneinsparende Ergänzung im diagnostischen Algorithmus der MS bieten. Sollte deren Effektivität reproduzierbar belegt werden, könnten Optische Kohärenztomographen -aufgrund der relativ zu Magnetresonanztomographen geringeren Anschaffungs- und Betriebskosten sowie der simpleren Handhabbarkeit-auch in strukturschwachen Gegenden in größerem Maßstab zum Einsatz kommen und somit Diagnosestellungs- und Behandlungschancen von an MS Erkrankten verbessern. 


\subsection{Fragestellung}

In der hier präsentierten Studie mit dem Titel „Retrospektive Evaluation retinaler Nervenfaserschichtdicke mit der cerebralen T2w-Läsionslast im MRT sowie dem Expanded Disability Status Scale (EDSS) bei pädiatrischen Patienten mit Multipler Sklerose" soll folgende Fragestellung untersucht werden:

Ist die Aussagekraft des OCT hinsichtlich Diagnostik und Verlaufsbeobachtung bei pädiatrischer MS mit der von MRT und EDSS vergleichbar?

Im Rahmen dieser Dissertation soll der mögliche klinische Nutzen der OCT als Erweiterung der aus neuroradiologischer Bildgebung, speziell der MRT, und klinischer Erfassung durch Klassifikationssysteme, wie dem EDSS, bestehenden Routinediagnostik der Multiplen Sklerose erörtert werden.

Um den möglichen Nutzen der OCT zu ermitteln, soll eine retrospektiv-vergleichende Betrachtung von kraniellen MRT-Datensätzen, welche die Objektivierung von MSLäsionsvolumina im Groß- und Kleinhirn zum Gegenstand haben, mit den OCTDatensätzen zur Atrophie der Nervenfaserschicht der Netzhaut, der sogenannten retinalen Nervenfaserschichtdicke (RNFLT), stattfinden. Die erhobenen Datensätze aus der OCT werden darüber hinaus einer Korrelation mit der u. a. mittels Expanded Disability Status Scale (EDSS) bestimmten Klinik der MS-Patienten zugeführt.

\subsection{Multiple Sklerose und deren gesellschaftliche Bedeutung}

Bei der Multiplen Sklerose beziehungsweise der Encephalomyelitis disseminata handelt es sich um eine in ca. $85 \%$ der Fälle schubförmig-remittierend verlaufende Autoimmunerkrankung (Atlas of MS 2013, Confavreux und Vukusic 2006), welche eine eindeutige Mehrbelastung des weiblichen Geschlechts aufweist (Whitacre 2001). Als weitere Verlaufsformen sind bei der adulten MS die primär-progrediente MS mit einem Anteil von ca. $10 \%$ und die progredient-schubförmige MS mit ca. 5\% bekannt (Atlas of MS 2013). Das durchschnittliche Ausbruchsalter der schubförmig verlaufenden Multiplen Sklerose liegt im frühen Erwachsenenalter bei ca. 30 Jahren und somit in einer Lebensphase, die für die Etablierung des sozio-ökonomischen Status der Patienten, im Speziellen für die Familien- und Karriereplanung, von weitreichender 
Bedeutung ist (Atlas of MS 2013). Der von der Multiple Sclerosis International Federation herausgegebene „Atlas of MS" von 2013 berichtet von einer Zunahme der weltweiten MS-Erkrankungen von 2,1 Millionen im Jahr 2008 auf 2,3 Millionen im Jahr 2013. Dies entspräche einem Zuwachs des globalen Medians der Prävalenz von 30/100.000 in 2008 auf 33/100.000 in 2013, wobei die Prävalenz für die Bundesrepublik Deutschland bei über 100/100.000 liegt. Darüber hinaus habe sich die Geschlechterverteilung der MS seit 2008 nicht-signifikant verändert und kann somit, auch für westliche Industrienationen wie die Bundesrepublik Deutschland, weitgehend unverändert mit einem Verhältnis von ca. 2:1 auf Seiten der weiblichen Bevölkerung angegeben werden (Atlas of MS 2013). Dieses Geschlechterverhältnis entspricht den von der Weltgesundheitsorganisation publizierten Daten (MS Atlas 2008). Auch in der Publikation von Whitacre konnte für die Multiple Sklerose eine klare Mehrbelastung des weiblichen Geschlechts aufgezeigt werden (Whitacre 2001). Publikationen von Jacobson et al. und Ansar Ahmed et al. legen nahe, dass das weibliche Geschlecht im Schnitt häufiger von Autoimmunerkrankungen betroffen ist als das männliche (Ansar Ahmed et al. 1985; Jacobson et al. 1997). Bezüglich der globalen Prävalenz lässt sich ein topografisches Muster erkennen: Mit zunehmender Entfernung vom Äquator beziehungsweise abnehmender Entfernung von den Erdpolen zeigt sich eine deutliche Zunahme der Prävalenz der MS (Pugliatti et al. 2006). Während die Prävalenz beispielsweise in Argentinien Schätzungen zufolge bei ca. 18/100.000 liegt, wird sie im äquatorial gelegenen Ecuador mit lediglich ca. 3,2/100.000 Einwohner beziffert (Atlas of MS 2013). Der höchste Wert für Europa wird mit 189/100.000 von Schweden erreicht (Atlas of MS 2013). Zu den einflussreichsten ätiologischen Faktoren der Multiplen Sklerose zählen laut Sadovnik et al. genetische sowie nicht-genetische bzw. Umweltfaktoren (Sadovnick et al. 1997). Bezüglich des genetischen Anteils der Ätiologie der MS kamen Ebers et al. zu dem Schluss, dass bei 19,8\% der 1044 geprüften MS-Patienten ein ebenfalls von MS betroffenes Familienmitglied aufzufinden war (Ebers et al. 2000). Besondere Aufmerksamkeit kommt dabei der Expression bestimmter Humanen-Leukozyten-Antigene (HLA) zu, speziell dem HLA DRB1*15 (Epplen et al. 1997; Compston 1999; Lin et al. 2012). Diese Sonderform erhöht das Risiko der Entwicklung einer MS um das 3- bis 4-Fache (Epplen et al. 1997). Während 75\% der an MS erkrankten Studienteilnehmer mindestens eine der HLA-Regionen 
DRB1*15,-16,-03 exprimierten, lag die Expressionsrate bei der gesunden Kontrollgruppe bei lediglich 47\% (Epplen et al. 1997).

Zur Historie der MS: Obgleich Jean-Martin Charcot (1825 - 1893) als Erstbeschreiber der Multiplen Sklerose als eigenständige Erkrankung gilt (Lublin 2005) und die Publikation, die ihm diese Beobachtung als Verdienst bescheinigt, auf das Jahr 1868 zurückreicht (Kumar et al. 2011), wurde bereits im späten 14. Jahrhundert eine Erkrankung beschrieben, welche nachträglich als Multiple Sklerose identifiziert werden konnte (Murray 2009). Diese Beobachtung basiert auf Aufzeichnungen aus den Vatikanischen Archiven, welche das Leben und die Symptome der Heiligen Ludwina von Schiedam beschreiben und das Auftreten erster Symptome auf deren 16. Lebensjahr datieren (Murray 2009).

\subsection{Multiple Sklerose bei Kindern}

Bis in die 1970er Jahre hinein galt das Auftreten von Multipler Sklerose bei Kindern als Rarität. Shaw und Alvord zufolge berichtet Kurtzke in seiner Publikation von 1970, dass „jegliche Erkrankung vor (Anmerkung des Autors: dem Alter von) 10 erst als letzter Ausweg als MS zu bezeichnen“ sei (zitiert nach: Shaw und Alvord 1987, Seite 252). Dem entgegen standen unter anderem die Publikationen von Shaw et al. aus dem Jahr 1987, welche die Multiple Sklerose bei Kindern zum Gegenstand hatten. Auch neuere Publikationen, wie beispielsweise die von Waubant et al., differenzieren zwischen verschiedenen Ausbruchsformen, der sogenannten adult-onset-MS und der earlyonset-MS, respektive dem Ausbruch der MS-Erkrankung im Erwachsenenalter und jenem im Kindesalter (Waubant et al. 2009). Zur Übertragbarkeit der Erkenntnisse zur adulten MS auf die kindliche, beispielsweise hinsichtlich der Behandlung, schreibt Hummel in ihrer Dissertation von 2015, dass es noch weitgehend unklar sei, ob eine Übertragbarkeit gegeben ist (Hummel 2015). Als Gründe hierfür führt sie Unterschiede in der Medikation und Diskrepanzen in der typischerweise erst im frühen Erwachsenenalter abgeschlossenen Myelinisierung an. Darüber hinaus spiele die im Kindesalter höhere Fähigkeit zur Remyelinisierung, welche den klinischen Krankheitsverlauf wesentlich beeinflussen kann, eine entscheidende Rolle. Der Publikation von Chitnis et al. zufolge, sind ca. 3\% der an Multiple Sklerose Erkrankten 
bei Manifestation ihrer ersten Symptome unter 18 Jahre alt (Chitnis et al. 2009). Der Atlas of MS von 2013 setzt den Anteil an MS-Patienten, welche bei Diagnosestellung unter 18 Jahre alt sind, bei ca. 2-5\% fest und berechnet eine gepoolte Inzidenz für pädiatrische MS von ca. 0,63/100.000 (Atlas of MS 2013). Darüber hinaus postuliert dieser, ähnlich wie Hummel 2015, dass pädiatrische und erwachsene MS-Patienten unterschiedliche Behandlung und Unterstützung benötigen (Atlas of MS 2013). Gemäß der Publikation von Ozakbas et al. liegt der Anteil an Patienten, welche bei Erstmanifestation ihrer MS-Erkrankung unter 16 Jahre alt sind, zwischen 1,2\% und 6\% (Ozakbas et al. 2003). Im Rahmen einer Studie an deutschen Patienten konnten Reinhardt et al. aufzeigen, dass die Inzidenz für pädiatrische MS in der Bundesrepublik Deutschland bei ca. 0,64 pro 100.000 Personenjahre liegt (Reinhardt et al. 2014). Des Weiteren konnte ein starker altersabhängiger Anstieg von 0,09 pro 100.000 Personenjahre bei Patienten unter 10 Jahren auf einen Wert von 2,64 pro 100.000 Personenjahre bei Patienten zwischen 14 und 15 Jahren verzeichnet werden (Reinhardt et al. 2014). Das Geschlechterverhältnis der pädiatrischen MS folgt der für die adulte MS geltenden Mehrbelastung des weiblichen Geschlechts nur teilweise. Duquette et al. zufolge liegt das Geschlechterverhältnis bei ca. 3:1 auf Seiten der weiblichen Studienteilnehmer (Duquette et al. 1987). Bioko et al. setzen in ihrer aktuelleren Studie aus dem Jahr 2002 das Geschlechterverhältnis bei 2,87:1 auf Seiten der weiblichen Studienteilnehmer fest (Boiko et al. 2002). Pohl berichtet bei der MS im Kindes- und Jugendalter von einem metaanalytisch bestimmten Geschlechterverhältnis von 2,3:1 auf Seiten der weiblichen MS-Patienten (Pohl 2007, Seite 13). Allerdings konnte sie, unter Verweis auf Hanefeld 1992, bei pädiatrischen MS-Patienten vor dem 11. Lebensjahr bzw. den sogenannten „true childhood“-MS-Patienten, metaanalytisch ein Geschlechterverhältnis von 0,9:1 auf Seiten der männlichen Patienten bestimmen (Pohl 2007, Seite 13; Hanefeld 1992). Das Geschlechterverhältnis bei pädiatrischer MS wird im Rahmen der Diskussion dieser Arbeit näher beleuchtet.

Der klinische Verlauf der pädiatrischen MS ist Renoux et al. zufolge in ca. $98 \%$ schubförmig-remittierend; bei Erwachsenen sei diese Verlaufsform in ca. 84\% der Fälle (bzw. 85\% der Fälle laut dem Atlas of MS 2013) zu beobachten (Renoux et al. 2007, Atlas of MS 2013). Sowohl bei Erwachsenen als auch bei Kindern wird der klinische Progress der Erkrankung mit Hilfe des sogenannten Expanded Disability Status Scale 
(EDSS) erhoben. Dieses zur Objektivierung der Symptomatik genutzte Klassifikationssystem wurde bereits im Jahr 1983 von John F. Kurtzke herausgegeben (Kurtzke 1983). Ein eigenes Klassifikationssystem für die pädiatrische MS existiert bislang nicht. Als wesentliches Charakteristikum der early-onset-MS kann die von Renoux et al. identifizierte durchschnittliche Latenz vom Erkrankungsbeginn bis zur sekundären Progression von ca. 28 Jahren angesehen werden (Renoux et al. 2007). Dieselbe Arbeitsgruppe schlussfolgert aus ihren gewonnenen Erkenntnissen, dass Patienten mit Multipler Sklerose des Kindesalters die Stadien der irreversiblen Behinderung langsamer erreichen als Patienten mit adult-onset-MS. Die beschriebenen Behinderungen manifestierten sich durch den früheren Ausbruch der Erkrankung allerdings in einem jüngeren Alter (Renoux et al. 2007). Besondere Aufmerksamkeit verdient auch die von Banwell und Anderson untersuchte neuropsychologische Leistungsfähigkeit von an Multipler Sklerose erkrankten pädiatrischen Patienten. Die Studie zeigte bei allen 10 Studienteilnehmern Einschränkungen der neuropsychologischen Leistungsfähigkeit (Banwell und Anderson 2005). Hummel zufolge seien bislang für Kinder und Jugendliche mit Multipler Sklerose keine spezifischen genetischen Risikofaktoren für die Entwicklung einer MS identifiziert worden und obgleich mehrere Umweltfaktoren, welche einen ätiologischen Einfluss auf die Entstehung der MS zu haben scheinen, identifiziert wurden, konnten diese nicht als für die MS spezifisch beschrieben werden (Hummel 2015). Als deutlicher Risikofaktor für die Entwicklung einer Multiplen Sklerose kann seit der Publikation von Levin et al. die Seropositivität für das Epstein-Barr-Virus (EBV) angesehen werden. In ihrer Studie konnte diese Arbeitsgruppe einen starken Zusammenhang zwischen der Entstehung der Multiplen Sklerose und der zeitgleichen Seropositivität für EBV identifizieren. Im Umkehrschluss kann die EBV-Seronegativität als protektiver Faktor für die Entwicklung einer Multiplen Sklerose angesehen werden (Levin et al. 2010). Die Studie mit 305 erkrankten Teilnehmern und einer 610 Personen starken Kontrollgruppe enthielt auch Patienten mit Multipler Sklerose in jugendlichem Lebensalter. Obgleich hier Levin et al. zufolge kein Kausalzusammenhang anzunehmen ist und infrage kommende Erklärungen angeboten werden, ist dennoch davon auszugehen, dass eine EBVInfektion als Risikofaktor für die Entstehung einer MS anzusehen ist (Levin et al. 2010). 
Zur Prognose der Multiplen Sklerose bei pädiatrischen Patienten schreiben Ghezzi et al., dass besonders in den ersten zwei Jahren nach Ausbruch der Krankheit mit einer erhöhten Anzahl an Schüben zu rechnen sei. Des Weiteren konnten sie aufzeigen, dass Behinderungen im ersten Krankheitsjahr stark mit dem Bestehen von Behinderungen im späteren Krankheitsverlauf korrelieren (Ghezzi et al. 2002). Patel et al. schreiben, dass, verglichen mit adulten Formen, die pädiatrische MS in den ersten zwei Krankheitsjahren zwar mit einer erhöhten Schubrate einhergeht, der Krankheitsprogress allerdings weniger fulminant verläuft (Patel et al. 2009).

\subsection{Optische Kohärenztomographie und Multiple Sklerose}

Bei der Optischen Kohärenztomographie handelt es sich um ein nicht-invasives, lichtbasiertes Verfahren zur Objektivierung physiologischer Prozesse und pathologischer Veränderungen der Strukturen der Makula sowie der Retina und ihrer einzelnen Schichten. Die OCT wird primär für Fragestellungen der Ophthalmologie eingesetzt, beispielsweise bei der hier zum Einsatz kommenden Messung der durchschnittlichen Nervenfaserschichtdicke der Netzhaut, der sogenannten Average Retinal Nerve Fiber Layer Thickness, kurz der Average RNFLT. Auch der Hersteller des in dieser Studie zum Einsatz gekommenen Optischen Kohärenztomographen CARL ZEISS MEDITEC führt in seinem Internetauftritt OCTs als Produkte für ophthalmologische Fragestellungen (Siehe Link zum Internetauftritt: http://www.zeiss.de/meditec/produkte/ophthalmologie/retina/diagnose/optische-

kohaerenztomographie/cirrus-familie/cirrus-hd-oct.html; Zugriff: 10.12.2016, 12:29Uhr).

In den vergangenen Jahren ist eine zunehmende Verwendung der Optischen Kohärenztomographie in anderen medizinischen Disziplinen, besonders der Neurologie bzw. der Neuroradiologie, zu beobachten. Bereits im Jahr 1999 konnten Parisi et al. aufzeigen, dass sich die OCT als geeignetes Mittel zum Nachweis der Korrelation zwischen der RNFLT und zuvor durchgemachter Optikusneuritis bei MS-Patienten erwies (Parisi et al. 1999). 
Bei der auch als Neuritis Nervi Optici (NNO) bekannten Optikusneuritis handelt es sich um eine Entzündung des Sehnervs, welche mit Symptomen wie starker Visusminderung und Augenbewegungsschmerz einhergeht. Hierbei kommt es Lamirel et al. zufolge zu einer Verdünnung der RNFLT am betroffenen Auge (Lamirel et al. 2010). Eine Vernachlässigung dieser Nebendiagnose könnte bei statistischer Betrachtung der Datensätze aus OCT und MRT bzw. OCT und klinischem Befund falschsignifikante Korrelationen verursachen, da die MS-bedingte RNFLT-Verdünnung nicht von der NNO-bedingten zu trennen ist.

Im Jahr 2010 wurde von Bock et al. geschlussfolgert, dass eine Verminderung der mittels OCT ermittelten RNFLT in allen vier Quadranten bei an MS erkrankten Patienten für die Existenz eines diffusen neurodegenerativen Prozesses spricht (Bock et al. 2010). Auch Dörr et al. konnten bei 104 an schubförmig-remittierender Multipler Sklerose erkrankten adulten Patienten im Alter zwischen 18 und 60 Jahren aufzeigen, dass die mittels OCT bestimmte RNFLT der 208 Augen mit der MS-bedingten Hirnatrophie korreliert. Daraus schlussfolgerten Dörr et al., dass die RNFLT als vielversprechender Parameter für die Evaluation der Neurodegeneration bei Multipler Sklerose anzusehen ist. Zur OCT im Speziellen postuliert die Arbeitsgruppe in ihrer Publikation, dass diese ein potentes Instrument zur Detektion MS-assoziierter retinaler Neurodegeneration ist (Dörr et al. 2011). Die Bedeutung der OCT als diagnostisches Mittel bei Multipler Sklerose und Optikusneuritis wurde von Kallenbach und Frederiksen in ihrer Publikation von 2007 sowie von Lamirel et al. in ihrer Publikation von 2010 diskutiert (Kallenbach und Frederiksen 2007; Lamirel et al. 2010). Lamirel et al. schlussfolgerten, dass die OCT zukünftig zu den Routinemaßnahmen bei Verlaufsbeobachtung, Messung von Krankheitsaktivität und Therapiekontrolle der Multiplen Sklerose zählen könnte (Lamirel et al. 2010).

Im Rahmen dieser Studie soll unter anderem die Relevanz der RNFLT als diagnostischer Parameter für die Multiple Sklerose bei pädiatrischen Patienten untersucht werden. Die OCT wurde von Schneider et al. im Rahmen ihrer Studie aus dem Jahr 2013 dazu verwendet, Unterschiede in der RNFLT-Atrophie bei Neuromyelitis optica und Multipler Sklerose aufzuzeigen. Im Ergebnis konnte für die Neuromyelitis optica eine stärkere Abnahme der RNFLT nachgewiesen werden als für die MS (Schneider et al. 2013). Aus diesen Erkenntnissen kann geschlussfolgert werden, dass Fortschritte in der 
OCT-Technologie die Möglichkeit der Differenzierung unterschiedlicher neurodegenerativer Prozesse eröffnen und die OCT somit im Diagnosealgorithmus derartiger Erkrankungen eine Rolle spielen könnte. Die Zuverlässigkeit der Bestimmung von RNFLT mit Optischen Kohärenztomographen wurde von Oberwahrenbrock et al. im Rahmen einer Metastudie untersucht, welche 27 Publikationen betrachtete. Im Resultat konnten Oberwahrenbrock et al. den zum Einsatz gekommen OCTs eine zuverlässige Wiederholbarkeit der ermittelten RNFLT für alle Retinaschichten mit Ausnahme der äußeren plexiformen Schicht bescheinigen (Oberwahrenbrock et al. 2015). Auch durch die Publikation der Ergebnisse einer multizentrischen Kohrtenstudie von Martinez-Lapiscina et al. wird die Bedeutung der Optischen Kohärenztomographie zur Beurteilung neurodegenerativer Erkrankungen hervorgehoben. Im Rahmen dieser Studie wurden unter anderem 664 an schubförmig-remittierender bzw. progredienter Multipler Sklerose erkrankte Patienten im Alter von $\geq 16$ Jahren auf die Ausprägung ihrer RNFLT-Atrophie untersucht. Die gewonnenen Ergebnisse lieferten laut Martinez et al. Belege dafür, dass die Messung der retinalen Nervenfaserschichtdicke durch Optische Kohärenztomographen für die Prognose des Aggravierungsrisikos der Multiplen Sklerose von relevantem Nutzen sei (Martinez-Lapiscina et al. 2016).

Da Potenzial und Nutzen der Optischen Kohärenztomographie für andere medizinische Fachdisziplinen nachgewiesen wurden, ist davon auszugehen, dass diese nicht länger ausschließlich für ophthalmologische Fragestellungen verwendet werden wird, sondern vielmehr eine Neudefinition ihrer Bedeutung für die Diagnostik und Verlaufsbeobachtung neurodegenerativer Erkrankungen, wie insbesondere der Multiplen Sklerose, erfahren wird.

\subsection{Magnetresonanztomographie und Multiple Sklerose}

Bei der Magnetresonanztomographie handelt es sich um ein nicht-invasives bildgebendes Verfahren, welches über den sogenannten Kernspin die Darstellung intrakorporaler Strukturen erlaubt. Beim Kernspin werden Protonen durch starke Magneten ausgerichtet bzw. durch starke Radiofrequenzimpulse angeregt. Dieses Verfahren kommt somit, anders als andere bekannte Verfahren wie die Röntgenbildgebung, szintigraphische Verfahren oder die Computertomographie, ohne 
die Verwendung radioaktiver Strahlung bzw. radioaktiver Strahler aus und kann somit als risikoarm eingestuft werden. Die Magnetresonanztomographie spielt in der Diagnostik der Multiplen Sklerose eine entscheidende Rolle. Da mit ihrer Hilfe die im ZNS lokalisierten, entzündlichen MS-typischen Läsionsherde identifiziert werden können, ist dieses diagnostische Instrument fest in die Diagnose- und Klassifikationskriterien der MS nach McDonald et al. und Paty et al. eingebunden (Paty et al. 1988; McDonald et al. 2001). Zur Diagnosestellung finden wesentliche Funktionsmodi des MRT Anwendung. So können gesuchte Läsionsherde beispielsweise mithilfe der 2 unterschiedlichen Wichtungseinstellungen identifiziert werden: Während die Inflammationsloki in der T1-Wichtung als sogenannte "black holes" imponieren, erscheinen sie in der T2-Wichtung als hyperintense Foci, wie die im Kapitel „Material und Methoden“ gezeigte Abbildung 1 darstellt. Obgleich diese nativen Aufnahmen den Eindruck vermitteln können, die Läsion relativ scharf von ihrer gesunden Umgebung abgrenzen zu können, ist der Einfluss sogenannter Normal Appearing White Matter (NAWM) nicht zu unterschätzen. Forschungen haben ergeben, dass die Ausdehnung bestimmter Läsionsherde ihre Sichtbarkeit mittels MRT überschreitet (Ge 2006). Bodini et al. konnten aufzeigen, dass eben diese mit dem MRT nicht zu erfassende NAWM bei der primär-progredienten Multiplen Sklerose maßgeblich an der Schädigung kortikaler Hirnareale beteiligt ist (Bodini et al. 2016). Zum Zwecke der genaueren Identifikation von Läsionsherden und deren Ausdehnung werden MRT-Aufnahmen von MS-Patienten häufig unter synchroner Applikation von Gadolinium-haltigem Kontrastmittel gefertigt. Das infundierte Kontrastmittel zeigt akut entzündete Herde, die eine Blut-Hirn-Schrankenstörung aufweisen. Darüber hinaus spielt in der MRT-basierten Diagnostik der MS die sogenannte Fluid-Attenuated Inversion Recovery-Sequenz, kurz FLAIR eine zentrale Rolle. Die Besonderheit dieser Sequenzen liegt in der Unterdrückung von MR-Signalen die von bestimmten Körperflüssigkeiten oder -geweben ausgehen. Die FLAIR-Sequenz unterdrückt im Speziellen die vom Liquor cerebrospinalis ausgehenden Signale. Der Nutzen dieser Sequenz in Diagnostik und Verlaufsbeobachtung der MS liegt in der präziseren Darstellbarkeit von periventrikulär gelegenen Läsionen, welche Adams et al. zufolge bei über $82 \%$ der MS-Patienten vorkommen (Adams et al. 1987). Diese hyperintens imponierenden Läsionen könnten aufgrund ihrer Lage in einer T2-gewichteten Sequenz 
von den ebenfalls hyperintensen MR-Signalen des Liquors teilweise überlagert und somit nur schwer abgegrenzt werden. Im Vergleich zur nativen T2-Wichtung bietet die FLAIR-Sequenz daher ein von diesem Störfaktor befreites alternatives Verfahren.

In der MRT-gestützten Diagnostik der MS spielt unter anderem die Läsionsmorphologie eine entscheidende Rolle, da Lage und Beschaffenheit fraglicher Läsionen Aufschluss über deren Krankheitswert liefern können. Wie oben bereits erwähnt, konnten Adams et al. aufzeigen, dass bei über 82\% ihrer 129 Patienten starken Kohorte periventrikulär gelegene Läsionsherde vorkamen (Adams et al. 1987). In diversen Studien konnte darüber hinaus die Relevanz von kortikal gelegenen Läsionsherden belegt werden (Kidd et al. 1999; Kutzelnigg und Lassmann 2005; Calabrese et al. 2008; Calabrese et al. 2010). Besondere Beachtung verdienen hierbei Studienergebnisse von Kutzelnigg und Lassmann, welche besagen, dass $26 \%$ der aktiven Läsionsherde kortikal bzw. juxtakortikal gelegen waren (Kutzelnigg und Lassmann 2005). Des Weiteren konnte in einer Studie der Arbeitsgruppe um Sinnecker aufgezeigt werden, dass 92\% der untersuchten Läsionsherde eine zentrale Vene aufwiesen (Sinnecker et al. 2012). Bei der Identifikation von MS-Läsionsherden ist darüber hinaus auch die in diversen Studien nachgewiesene Existenz eines hypointensen Randsaums zu beachten (Llufriu et al. 2010; Sinnecker et al. 2012). Die Läsionsmorphologie ist Kuchling et al. zufolge in der primär-progredienten und in der schubförmig-remittierenden MS weitestgehend identisch (Kuchling et al. 2014). Bei Kindern konnten die läsionsmorphologischen Zusammenhänge für kortikale Läsionen nicht in demselben Ausmaß nachgewiesen werden wie bei adulten MS-Patienten (Absinta et al. 2011), obgleich Polman et al. im Rahmen der Revision der McDonald-Kriterien postulieren, dass Kinder häufig MSLäsionen in vier typischen Lokalisationen aufweisen. Demnach finden sich Läsionsherde besonders häufig periventrikulär, juxtakortikal, im Hirnstamm sowie im Rückenmark (Polman et al. 2011). Als Unterschied in der Läsionsmorphologie bei MSPatienten in einem Alter unter 11 Jahren im Vergleich zu solchen in der Adoleszenz kann festgehalten werden, dass jüngere Patienten häufiger weniger definierte und eher konfluierende Läsionsherde aufweisen als ältere Patienten (Chabas et al. 2008).

Trotz dieser MRT-gestützten relativ eindeutigen Bildgebung sind die Läsionsherde wie viele über bildgebende Verfahren identifizierbare Pathologien letztlich das Produkt der Interpretation des Diagnostikers und somit nicht zweifelsfrei als Läsionsherde zu 
deklarieren. Zur definitiven Diagnosestellung der MS sind daher weitere Diagnosetechniken, wie beispielsweise die klinische Erfassung der Symptomatik über den sogenannten EDSS, anzuwenden. Auch Filippi et al. beschäftigen sich mit der Präzisierung der Diagnostik der MS mittels MRT. Besonderes Augenmerk wurde dabei auf die Abgrenzung der Multiplen Sklerose von ähnlich imponierenden Erkrankungen sowie auf die Fortschritte in der MRT-Technologie und deren Nutzen für die Diagnostik gelegt (Filippi et al. 2016). Das Resultat ist eine Liste mit Modifikationsempfehlungen für die bereits von Polman et al. modifizierten Diagnose- und Klassifikationskriterien nach McDonald (McDonald et al. 2001; Polman et al. 2011). Von besonderer Bedeutung bei der Diagnose der Multiplen Sklerose durch MRT ist die Existenz des sogenannten „Klinisch-Radiologischen Paradoxons“ (Barkhof 2002). In dieser Zeit ist eine Identifikation krankheitstypischer Pathologien im MRT, speziell der häufig paraventrikulär anzutreffenden Läsionsherde, nicht möglich (Barkhof 2002). 


\section{Material und Methoden}

Im nachfolgenden Kapitel werden die Zusammenstellung der untersuchten Patientenkohorte sowie die zum Einsatz gekommenen diagnostischen Instrumente erklärt. Darüber hinaus soll der Prozess der Datenaquisition und -auswertung näher beschrieben werden.

\subsection{Studiendesign und Auswahlkriterien}

Im Rahmen der retrospektiv vergleichenden Studie zur MS bei pädiatrischen Patienten wurde die Evaluation bestehender Datensätze hinsichtlich der Vergleichbarkeit der Aussagekraft von MRT und OCT in Bezug auf Nervenfaseratrophie bei an MS erkrankten Kindern vorgenommen. Korrelationen mit der Klinik der Patienten wurden u. a. mithilfe des EDSS, welcher MS-typische klinische Ausfallerscheinungen misst, untersucht. Die Ethikkommission der UMG äußerte an dieser Studie keine Bedenken.

Die Datensätze aller in die Studie eingeschlossenen Patienten mussten den folgenden Ein- bzw. Ausschlusskriterien entsprechen:

Einschlusskriterien:

1.) In dieser Studie wurden MS-Patienten der Kinderklinik der Universitätsmedizin Göttingen berücksichtigt, welche zum Zeitpunkt der Diagnosestellung nicht älter als 19 Jahre alt waren.

2.) Als Einschlusskriterium für die MR-Aufnahmen wurde das Vorliegen einer an der UMG mit nachfolgend beschriebenem Gerät angefertigten, T2-gewichteten Sequenz des Gehirns in transversaler Orientierung mit einer Schichtdicke von 4 Millimetern ( $\mathrm{mm})$ definiert.

3.) Als Einschlusskriterium für die OCT-Untersuchungen galt das Vorliegen zweier an der Augenklinik der UMG konsekutiv durchgeführter Untersuchungen, wobei jeweils eine die RNFLT (in $\mu \mathrm{m}$ ) des rechten und die andere die des linken Auges erfasste. 
4.) Bezüglich der klinischen Parameter galt die Dokumentation von EDSS, Schubsumme und Krankheitsdauer für die jeweiligen Patienten als Einschlusskriterium.

Ausschlusskriterium:

Da in dieser Studie möglichst der separate Einfluss von MS auf die RNFLT bestimmt werden soll, mussten die Augen, an denen im Krankheitsverlauf eine NNO dokumentiert wurde, von der weitergehenden Betrachtung ausgeschlossen werden.

Den oben formulierten Einschlusskriterien für MRT, OCT und Klinik entsprachen 60 von 65 zunächst näher betrachteten Patienten. Von diesen wurden 7 Patienten mit beidseitiger NNO sowie 8 Patienten mit unklarer NNO-Dokumentation von der weitergehenden Betrachtung ausgeschlossen, sodass die endgültige Auswertung mit den Datensätzen von 45 Patienten erfolgte.

\subsection{Die Optische Kohärenztomographie (OCT)}

Die OCT-Untersuchungen wurden bei allen 45 in die Studie eingeschlossenen Patienten unter identischen Aufnahmebedingungen in der Augenklinik der Universitätsmedizin Göttingen durchgeführt. Bei dem verwendeten Gerät handelte es sich um einen von CARL ZEISS MEDITEC hergestellten Optischen Kohärenztomographen der Baureihe CIRRUS HD-OCT (4000-3300). Bei der installierten Software handelte es sich um die Version 7.0.1.286.

\subsection{Die Magnetresonanztomographie (MRT) und Aufnahmetechnik}

Die MR-Aufnahmen der 45 eingeschlossenen Studienteilnehmer wurden an der Universitätsmedizin Göttingen nach einheitlichen Sequenzparametern und Schichtführungen an einem 3 Tesla MRT der Baureihe MAGNETOM TIM Trio des Herstellers SIEMENS unter Verwendung der Betriebssystemversion Syngo MR B13 erstellt. Die Aufnahmen unterschieden sich in 4 Fällen lediglich im Parameter Repetition Time. Hierbei handelt es sich um die Zeitspanne zwischen der Applikation zweier Pulse auf dieselbe Sequenzschicht, welche bei der Aufnahmedauer eine 
wesentliche Rolle spielt (http://www.fonar.com/glossary.htm; Zugriff: 10.12.2016, 12:27Uhr). Bei der für die Sequenzerstellung verwendeten Spule handelt es sich um eine in der klinischen Routine verwendete 12-Kanal-Kopf-Spule mit der Produktbezeichnung „3T Head MATRIX, A Tim Coil“. Die jeweils 35 (bzw. in einem Fall 38) Schichten umfassenden Sequenzen wurden nicht eigens für die Studie erstellt, sondern aus einem Pool von Routineuntersuchungen ausgewählt.

\subsection{Verwendete Bild-Analyse-Software}

Zur softwaregestützten Analyse der T2-gewichteten MR-Sequenzen, namentlich der Quantifizierung der Gesamtzahl an individuellen entzündlichen MS-Läsionen in Zerebrum und Zerebellum pro Patient sowie der Volumenbestimmung dieser Herde, wurden die pseudonymisierten Datensätze u. a. in die frei verfügbare Medical Image Processing, Analysis and Visualisation Software eingespeist. Bei der genutzten Ausführung handelte es sich um die Version mit der Bezeichnung v6.0.1 (MIPAV, Version 6.0.1, National Institute of Health, Bethesda, MD, USA). Zur Aufbereitung der ausgewählten Sequenzen bzw. der Sequenz-Parameter wurde die Bilddatenmanagement-Software Extensible Neuroimaging Archive Toolkit, kurz XNAT, der Neuroinformatics Research Group (Marcus et al. 2007), verwendet (XNAT, Versionen 1.6.2, 1.6.3 und 1.6.5, NRG, Washington University School of Medicine, St. Louis, MO, USA).

Zur Erfassung der jeweiligen Region of Interest (ROI) wurden die einzelnen in der T2Wichtung hyperintens erscheinenden MS-Läsionen, die auf eine Demyelinisierung hinweisen, mit der MIPAV-Software unter Zuhilfenahme der Auswahlwerkzeuge auf den einzelnen Sequenzschichten markiert. Dadurch konnte zum einen die individuelle Läsionssumme bestimmt, zum anderen eine dreidimensionale Läsionsmaske erstellt werden, welche dabei half, die Summe an markierten ROls zu einem sogenannten Volume of Interest (VOI) zusammenzufassen. Dazu wurden die neu gewonnenen Läsionsmasken anschließend in die FMRIB Software Library, kurz FSL, zur Volumenbestimmung eingespeist (FSL, Version 5.0.7, Analysis Group, FMRIB, Oxford, GB). Die berechneten Läsionsvolumina werden im Folgenden in Kubikmillimeter $\left(\mathrm{mm}^{3}\right)$ angegeben. 
Nachfolgend ist beispielhaft ein mittels Magnetresonanztomographie erstelltes Schnittbild aus einer der zur Datenerhebung verwendeten, T2-gewichteten nativen Sequenzen abgebildet. Die roten Umrandungen folgen dabei den sichtbaren Grenzen der auf dieser Schicht zur Darstellung gekommenen Einzelläsionen. Zur besseren Darstellbarkeit in dieser Arbeit wurden u. a. Helligkeit und Kontrast von Abbildung 1 mit dem Fotos Programm des Windows-Computerbetriebssystems angepasst (Fotos, Windows 10, Microsoft).

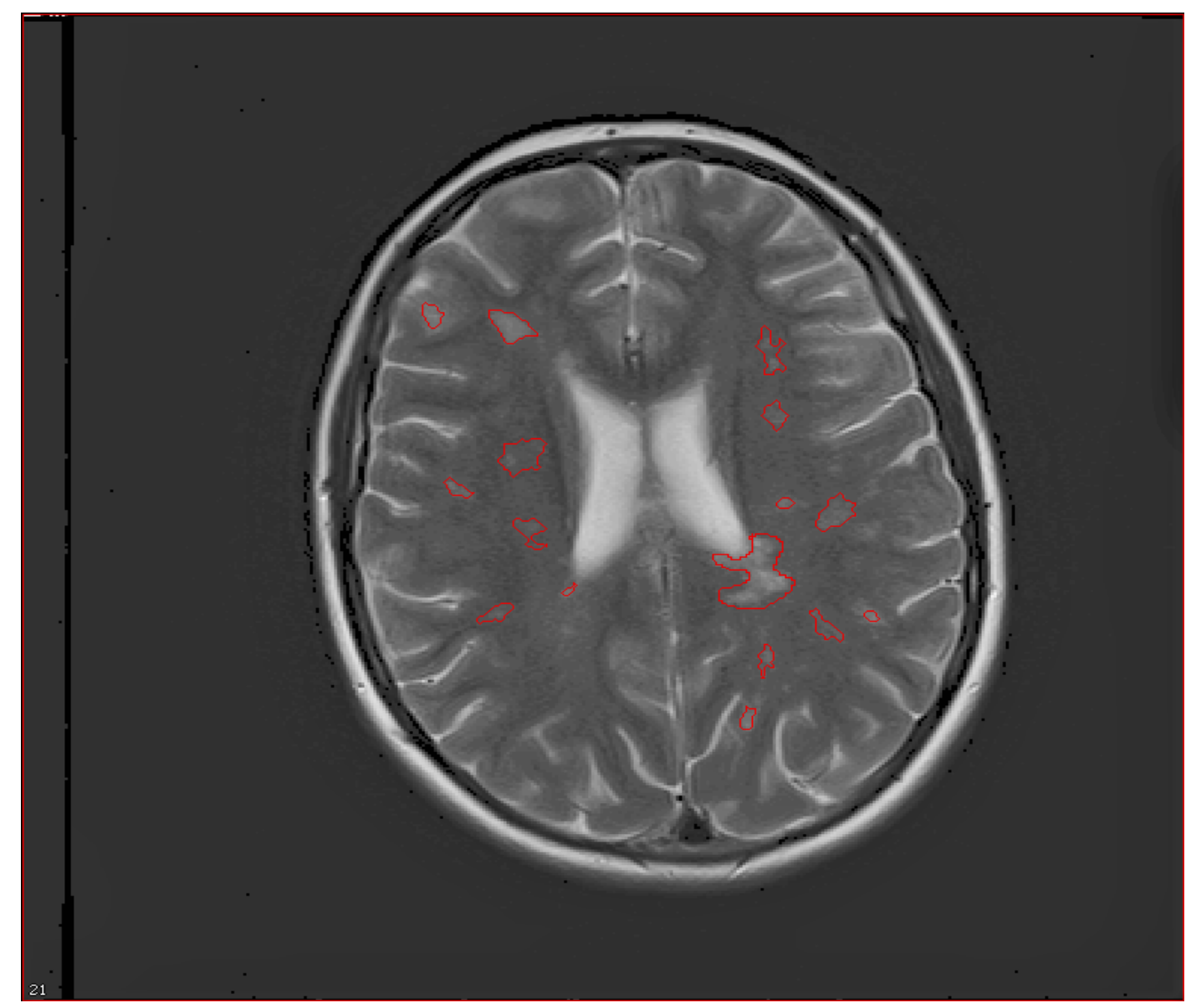

Abbildung 1: Läsionsherde im MRT

Anschließend wurden Schnittbilder wie das obige in eine Läsionsmaske zur Quantifizierung der individuellen Gesamtvolumina der MS-typischen Läsionsherde überführt. Die folgende Abbildung zeigt dieselbe Schicht, wie sie in Abbildung 1 zur Darstellung kommt, nach ihrer Überführung in die Läsionsmaske. 


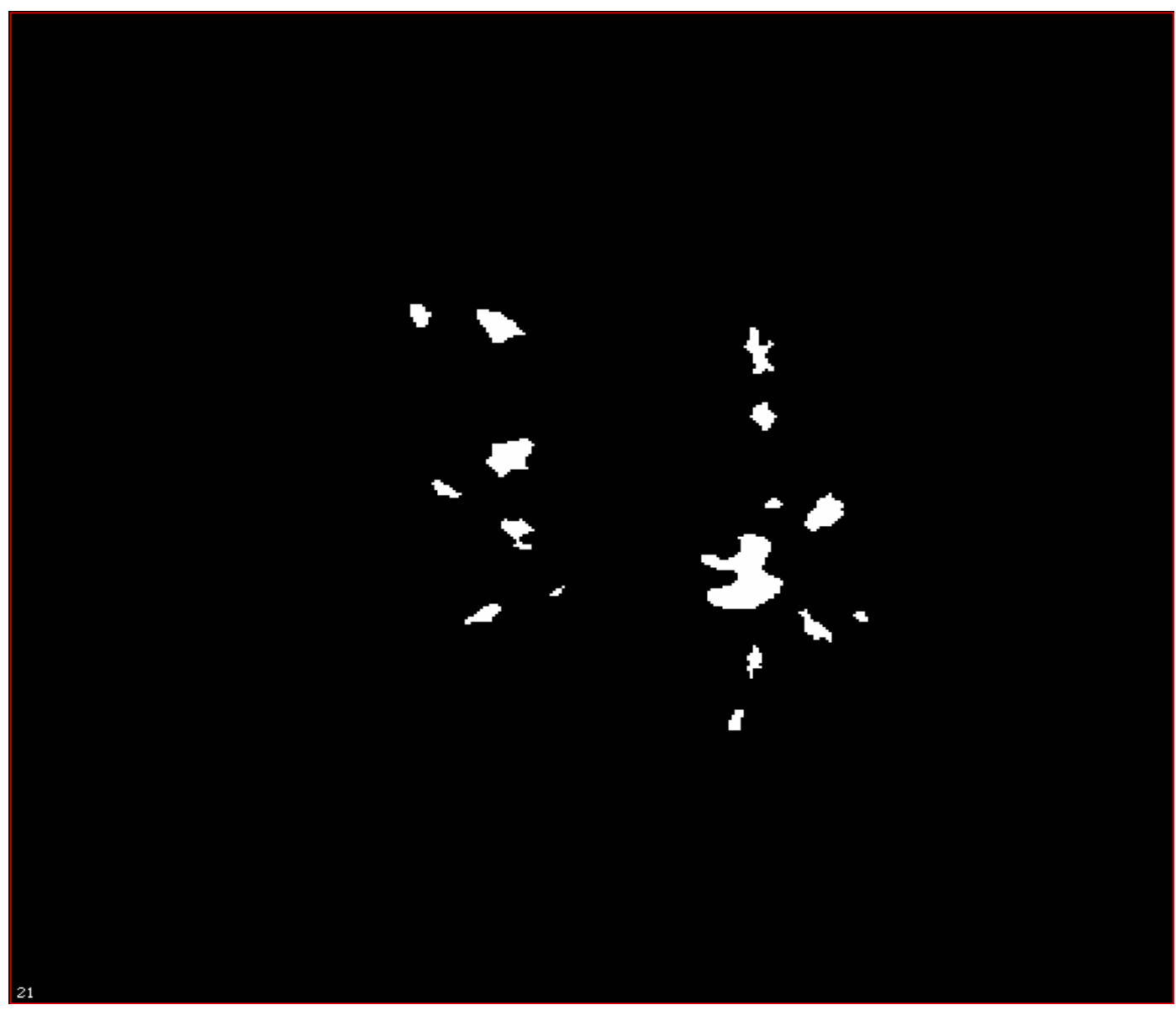

Abbildung 2: Läsionsmaske

\subsection{Klinische Untersuchungen}

Der Krankheitsverlauf der hier betrachteten Patienten wurde an der UMG im longitudinalen Verlauf durch klinische neuropädiatrische Parameter erfasst und dokumentiert. Die in unregelmäßigen Abständen erfolgenden, engmaschigen Routineuntersuchungen umfasste hierbei die Dokumentation der zu beobachtenden Schübe und deren zeitliche Einordnung in die Krankheitsgeschichte. Zur Messung MStypischer, klinisch-neurologischer Defizite und Ausfallerscheinungen wird darüber hinaus im Rahmen der Routineuntersuchungen der 1965 vom US-amerikanischen Neurologen John F. Kurtzke entworfene und 1983 überarbeitete Expanded Disability Status Scale (EDSS) erhoben. Im Rahmen dieser Untersuchung wird die Beeinträchtigung diverser funktioneller Systeme des Körpers dokumentiert. Die folgende Einteilung ist Kurtzkes Publikation mit dem Titel „Rating neurological 
impairment in MS: An Expanded Disability Status Scale (EDSS)" entnommen: Die sieben funktionellen Systeme umfassen hierbei Pyramidenbahnfunktionen, Zerebelläre Funktionen, Hirnstammfunktionen, Sensoriumsfunktionen, Darm- und Blasenfunktionen, Visuelle (oder Optische) Funktionen, Zerebrale (oder Mentale) Funktionen sowie Andere Funktionen (Kurtzke 1983, Seite 1450-1451). Diese funktionellen Systeme werden proportional zum Grad der Beeinträchtigung aufsteigend mit einem Wert von Grad 0 bis 5 bzw. 0 bis 6 angegeben. Eine Ausnahme bildet diesbezüglich der Unterpunkt Andere Funktionen, hier wird nur zwischen den Graden 0 und 1 unterschieden, wobei 0 für „Keine“ und 1 für „Alle anderen neurologischen Befunde, die auf die MS zu attribuieren sind (spezifizieren)“ steht (Kurtzke 1983, Seite 1451). Die EDS-Skala rangiert hierbei zwischen den Graden 0 bis 10, wobei Grad 0 den vergebenen Wert für das Vorliegen normaler neurologischer Funktionen und Grad 10 den Wert für einen MS-bedingten Tod umschreibt.

\subsection{Statistische Auswertung}

Die Archivierung der individuellen Untersuchungsergebnisse von MRT und OCT, hier sowohl Summe und Volumen der intrakraniellen Läsionen als auch die RNFLT und die Ergebnisse der klinischen Untersuchungen, hier die Summe durchgemachter MSSchübe, der erhobene EDSS und die Krankheitsdauer, erfolgte in einer Übersichtstabelle. Diese wurde mit dem Tabellenkalkulationsprogramm Microsoft Office Excel 2007 erstellt (Excel 2007, Microsoft). Mit den dazugehörigen Kalkulationswerkzeugen wurden zur weiteren Betrachtung Berechnungen, unter anderem von Mittelwerten und Standardabweichungen, vorgenommen. Die statistische Auswertung erfolgte darüber hinaus unter Zuhilfenahme des Statistik- und Analyseprogramms STATISTICA von Dell. Bei der verwendeten Ausführung handelt es sich um die Version 12 (STATISTICA, Version 12, Dell). Als Signifikanzniveau wurde ein $p$ von $<0,05$ festgelegt. Stärkere Signifikanzen wurden mit einem $\mathrm{p}$ erfasst, welches bei einem Wert von $<0,01$ als sehr bzw. bei einem von $<0,001$ als hoch signifikant gilt. Die Korrelationsmessung erfolgte mittels des Rangkorrelationskoeffizienten nach Charles Spearman, dem Spearmans Rho. Dieser Rangkorrelationskoeffizient kam bei der hier vorgenommenen explorativen Studie zum Einsatz, da er, anders als der 
Korrelationskoeffizient nach Pearson, keinen linearen Zusammenhang verglichener Werte unterstellt. Darüber hinaus wurde Spearmans Rho aufgrund seiner geringeren Anfälligkeit gegenüber stark abweichenden Einzelwerten, welche auch als Ausreißer bezeichnet werden, dem Korrelationskoeffizienten nach Pearson vorgezogen. Die Korrektur der ermittelten Ergebnisse für multiples Testen erfolgte nach der BonferroniHolm-Methode. 


\section{Ergebnisse}

Im Folgenden werden die ermittelten Ergebnisse der Datenauswertung im Detail präsentiert.

\subsection{Epidemiologie und Aufbau der Kohorte}

Für 52 von 60 den Einschlusskriterien entsprechenden Patienten bzw. 104 Augen lag eine Dokumentation der NNO-Anamnese vor. Hierbei bestand bei 29 Patienten keine NNO (nNNO) in der Anamnese, bei 16 Patienten bestand mindestens eine unilaterale NNO, bei den übrigen 7 Patienten waren im Verlauf beide Augen von mindestens einer NNO betroffen. Es ergibt sich ein Verhältnis von nNNO- zu NNO-Patienten von 74 zu 30 Augen.

Wie der nachfolgenden Grafik zu entnehmen ist, konnten die von Trip et al. gemachten Beobachtungen hinsichtlich der stärkeren Verdünnung der RNFLT bei MS-Patienten mit NNO-Anamnese im Verhältnis zu solchen ohne NNO-Anamnese für die hier betrachteten Augen reproduziert werden (Trip et al. 2005).

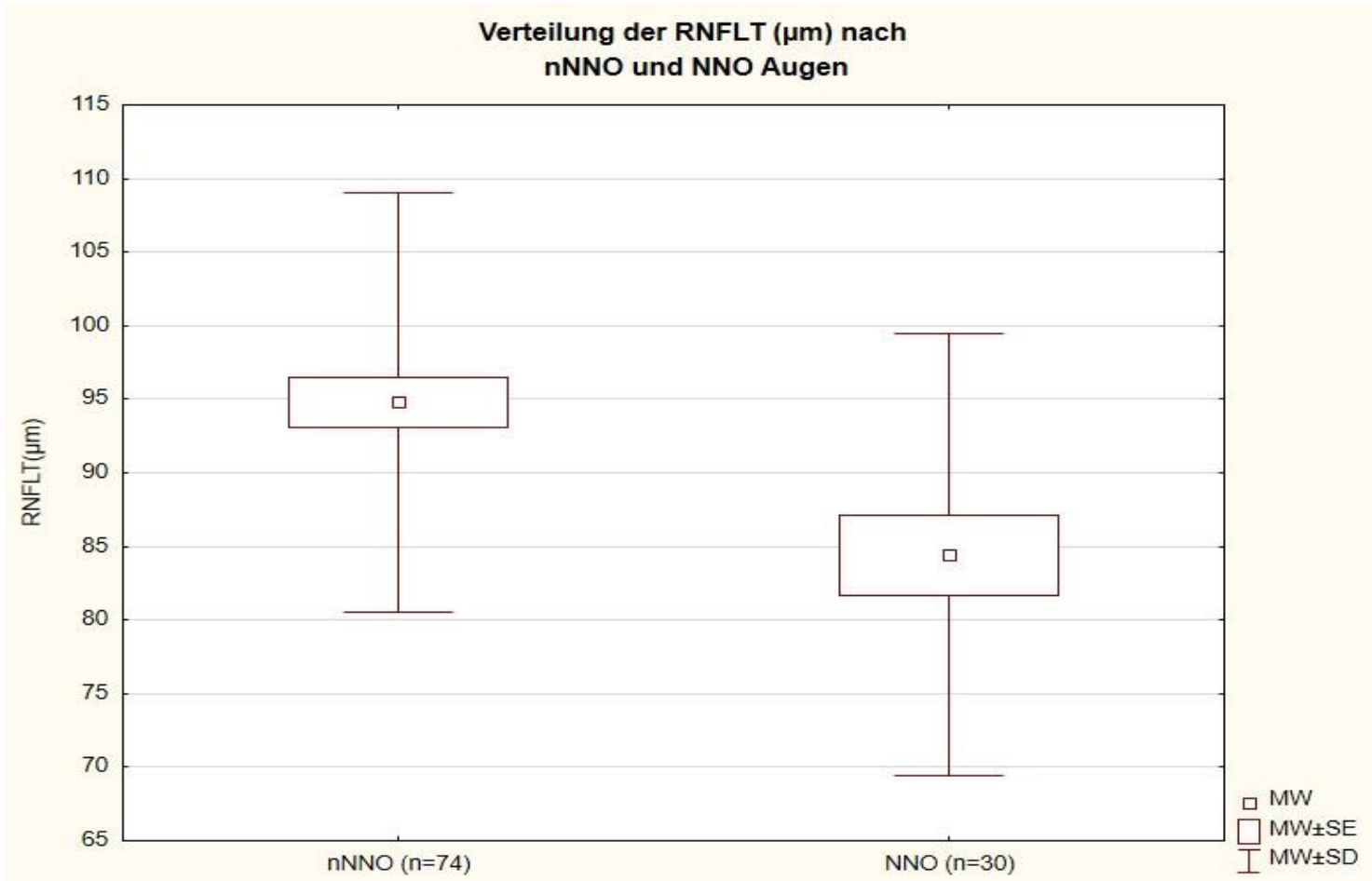

Abbildung 3: Verteilung der RNFLT $(\mu \mathrm{m})$ nach nNNO und NNO Augen 
Durchschnitt und Standardabweichung der RNFLT der nNNO-Augen lagen bei $94,79 \pm$ $14,2 \mu \mathrm{m}(\mathrm{MW} \pm \mathrm{SD})$, die Werte für die von mindestens einer NNO betroffenen Augen betrugen $84,43 \pm 15,03 \mu \mathrm{m}(\mathrm{MW} \pm \mathrm{SD})$.

Abbildung 3 veranschaulicht, dass für die hier ausgeschlossenen NNO-Augen eine im Durchschnitt ca. $10 \mu \mathrm{m}$ dünnere RNFLT bestimmt werden konnte und ein Einschluss dieser Augen die Ergebnisse entsprechend verfälschen würde.

Für die weitergehende Betrachtung des MS-Einflusses auf die RNFLT wurden daher die 7 Patienten mit bilateraler NNO in der Anamnese sowie die betroffenen Augen der 16 Patienten mit unilateraler NNO ausgeschlossen.

Da für die 16 Patienten mit unilateraler NNO nur das kontralaterale nNNO-Auge betrachtet werden kann, wurde für die 29 Patienten ohne NNO in der Anamnese aufgrund der geringen interokulären Differenz der RNFLT, welche der nachfolgenden Grafik zu entnehmen ist, aus den RNFLT-Werten beider Augen ein Mittelwert gebildet und für die weitergehende Betrachtung verwendet.

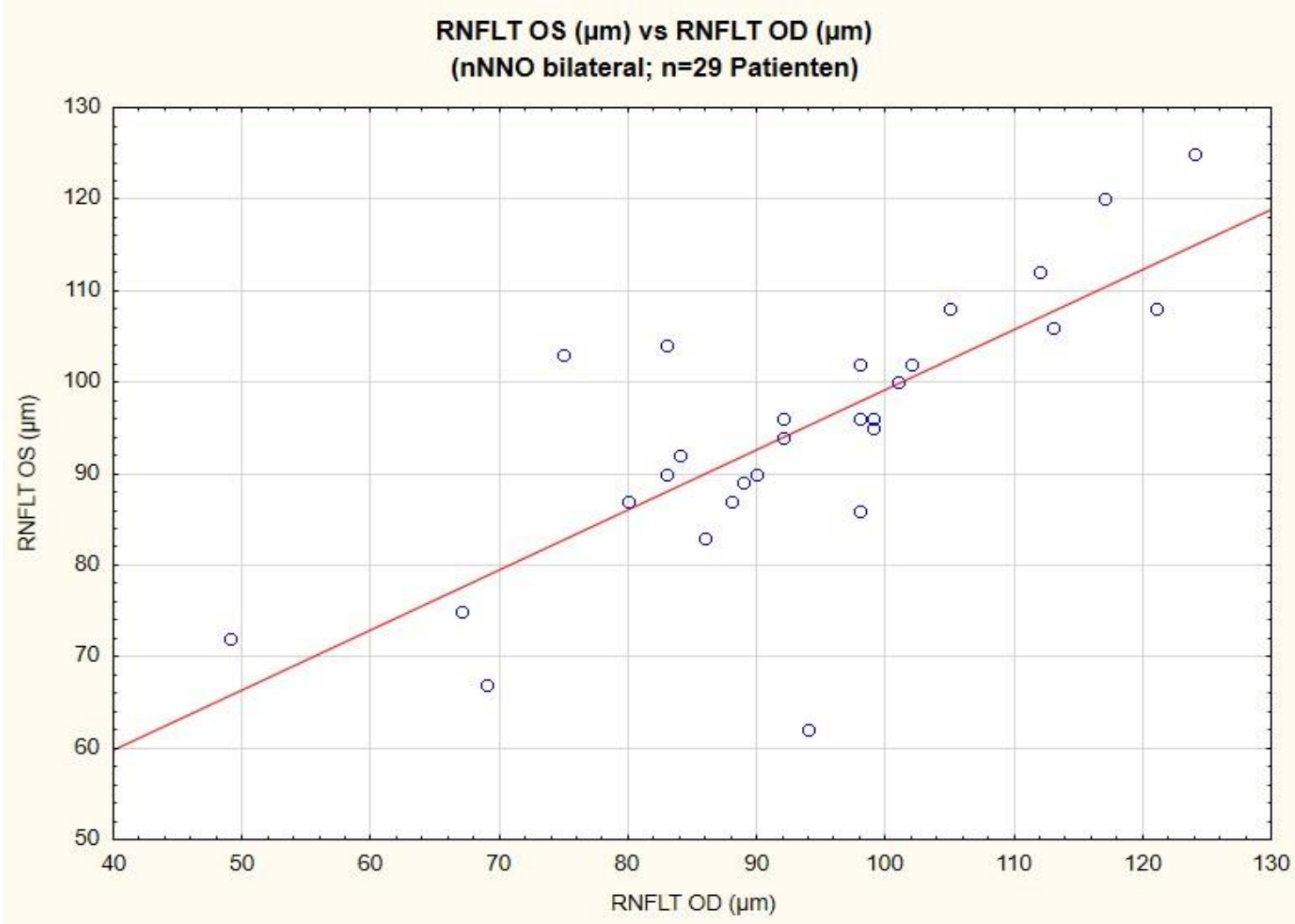

Abbildung 4: RNFLT OS ( $\mu \mathrm{m})$ vs. RNFLT OD $(\mu \mathrm{m})$; (nNNO bilateral; $\mathrm{n}=29$ Patienten) 
Für die statistische Auswertung wurde daher je ein Datensatz für 45 Patienten, welche einander hinsichtlich MR-Aufnahmebedingungen und MR-Aufnahmequalität weitgehend entsprachen, betrachtet. Aufgrund der Zusammenstellung der Kohorte nach zufälliger Übereinstimmung der Ein- und Ausschlusskriterien wurden auf Subgruppenvergleiche nach Alter bzw. nach Geschlecht verzichtet.

Die Altersverteilung lag hierbei zum MR-Sequenzaufnahmezeitpunkt zwischen 9,75 und 19 Jahren bei einem Durchschnittsalter von 16,33 Jahren. Das Verhältnis von weiblichen zu männlichen Studienteilnehmern lag bei 31 zu 14. Die Aufnahmezeitpunkte von MRT und OCT lagen im Maximum 365 Tage auseinander und erfolgten im Minimum am selben Tag, wobei das Durchschnittsintervall zwischen den Untersuchungszeitpunkten $67 \pm 109$ (MW \pm SD) Tage betrug, der Median lag hier bei einem Tag. Die zeitliche Differenz zwischen dem Aufnahmezeitpunkt der MRSequenzen und der Erhebung des EDSS lag im Maximum bei 309 Tagen und erfolgte im Minimum einen Tag später, das Durchschnittsintervall zwischen beiden Messpunkten lag bei $20 \pm 57(\mathrm{MW} \pm$ SD) Tagen, der Median betrug hier einen Tag. Auch die Parameter Schubsumme und Krankheitsdauer orientieren sich am MR-Aufnahmezeitpunkt. Die nachfolgende Übersichtstabelle soll die von den Studienteilnehmern erhobenen und nachfolgend hinsichtlich ihrer Korrelation mit der RNFLT untersuchten Datensätze veranschaulichen.

Tabelle 1: Struktur der Kohortendaten

\begin{tabular}{|l|l|l|r|r|}
\hline Parameter & MW & Skala & Median & SE \\
\hline RNFLT $(\mu \mathrm{m})$ & $95,41 \pm 12,71$ & $60,5-124,5$ & 96 & 1,89 \\
\hline Läsionsvolumen $\left(\mathrm{mm}^{3}\right)$ & $10716,7 \pm 14337$ & $825,1-84645,2$ & 6434,6 & 2137,2 \\
\hline Läsionssumme & $42,1 \pm 33,9$ & $5-145$ & 32 & 5,05 \\
\hline Alter (Jahre) & $16,33 \pm 1,91$ & $9,75-19$ & 16,75 & 0,285 \\
\hline Krankheitsdauer (Monate) & $31,6 \pm 26,3$ & $0-140$ & 28 & 3,92 \\
\hline Schubsumme & $3,1 \pm 2,2$ & $1-10$ & 3 & 0,33 \\
\hline EDSS & & $0-3,5$ & 0 & \\
\hline
\end{tabular}




\subsection{Korrelationen}

Die Korrelation erfolgte im Allgemeinen für die 45 Studienteilnehmer umfassende Gesamtkohorte. Da sich die erhobenen Daten für eine parametrische Korrelation nach Pearson als zu heterogen erwiesen, erfolgte die Korrelationsmessung mittels des Rangkorrelationskoeffizienten nach Charles Spearman, dem sogenannten Spearmans Rho. Das minimale Signifikanzniveau wurde auf $p<0,05$ festgesetzt; stärkere Korrelationen mit einem $p<0,01$, bzw. $p<0,001$ werden gesondert hervorgehoben. Diese Korrelation umfasst im Einzelnen die sieben in den Kapiteln 3.2 bis einschließlich 3.5 dokumentierten Parameter, namentlich der Untersuchung von RNFLT, der Untersuchung von Läsionsvolumina und Einzelläsionssumme, der klinischen Untersuchungen des EDSS und der Schubsumme sowie der Betrachtung von Krankheitsdauer und Alter. Die gefundenen Korrelationen sind nachfolgend sowohl in Textform als auch tabellarisch dokumentiert. Aus Gründen der Übersichtlichkeit beschränken sich alle Angaben, mit Ausnahme derer der p-Werte, auf maximal zwei Dezimalstellen. Die nachfolgende Tabelle zeigt die Ergebnisse der durchgeführten Korrelationsberechnungen. Die Angaben umfassen sowohl die Korrelationskoeffizienten und naiven $\mathrm{p}$-Werte als auch die nach der Bonferroni-HolmKorrekturmethode für multiples Testen adjustierten $p$-Werte.

Tabelle 2: Korrelationen

\begin{tabular}{|c|c|c|c|}
\hline $\begin{array}{l}\text { Spearman Rho-Rangkorrelation } \\
\text { (Signifikanzniveau }<0,05 ; *=p<0,05 ; \\
* *=p<0,01 ; * * *=p<0,001 \text { ) }\end{array}$ & $\begin{array}{l}\text { Korrelations- } \\
\text { koeffizient }\end{array}$ & $\begin{array}{l}\text { p-Wert } \\
\text { (Naiv) }\end{array}$ & $\begin{array}{l}\text { p-Wert } \\
\text { (Bonferroni-Holm) }\end{array}$ \\
\hline RNFLT vs. Läsionsvolumen & $-0,492$ & $0,0006 * * *$ & $0,0036 * *$ \\
\hline RNFLT vs. Schübe & $-0,432$ & $0,003034 * *$ & $0,01517^{*}$ \\
\hline RNFLT vs. Krankheitsdauer & $-0,387$ & $0,008712 * *$ & $0,034848^{*}$ \\
\hline RNFLT vs. EDSS & $-0,347$ & $0,019385^{*}$ & 0,058155 \\
\hline RNFLT vs. Läsionssumme & $-0,344$ & $0,020742 *$ & $0,041483^{*}$ \\
\hline RNFLT vs. Alter & $-0,097$ & 0,525311 & 0,525311 \\
\hline
\end{tabular}




\subsection{Ergebnisse der OCT-Untersuchungen}

Zur Objektivierung der Nervenfaserschicktdicke des Nervus opticus, der sogenannten Retinal Nerve Fiber Layer Thickness (RNFLT), wurde der unter 2.2 beschriebene Optische Kohärenztomograph verwendet. Mithilfe dieses Geräts konnte die Nervenfaserschichtdicke in Mikrometern $(\mu \mathrm{m})$ ausgedrückt und somit eine etwaige, im Krankheitsverlauf der Multiplen Sklerose aufgetretene Nervenfaseratrophie dokumentiert werden. Im Folgenden werden die gewonnenen Ergebnisse für die 45 Patienten dargelegt. Für die Studienteilnehmer lag die RNFLT im Durchschnitt bei $95,41 \pm 12,71 \mu \mathrm{m}(\mathrm{MW} \pm \mathrm{SD})$. Die bei Betrachtung der Gesamtkohorte gemessene maximale Nervenfaserschichtdicke lag bei $124,5 \mu \mathrm{m}$ bei einem gemessenen minimalen Durchmesser von 60,5 $\mu \mathrm{m}$. Der berechnete Median lag bei $96 \mu \mathrm{m}$.

Da im Rahmen dieser Studie aufgezeigt werden soll, inwiefern die mittels OCT ermittelete RNFLT in den Diagnosealgorithmus der MS intergriert werden kann, werden in den nachfolgenden Kapiteln u. a. die in Tabelle 2 zusammengefassten Korrelationen der RNFLT mit den übrigen betrachteten Parametern im Detail dokumentiert.

\subsection{Ergebnisse hinsichtlich der MRT-Untersuchungen}

\subsubsection{Läsionsvolumen}

Zur Objektivierung des Gesamtvolumens wurden die einzelnen in der T2-Wichtung hyperintens erscheinenden Läsionsherde als sogenannte Regions of Interest (ROIs) klassifiziert. Von diesen wurden Gesamtzahl und Gesamtvolumen bestimmt. Unter den 45 Studienteilnehmern konnte ein durchschnittliches aufaddiertes Läsionsvolumen von $10716,7 \pm 14337 \mathrm{~mm}^{3}$ (MW \pm SD) bestimmt werden. Von den gemessenen Werten für Läsionsvolumina lag das Maximum bei $84645,2 \mathrm{~mm}^{3}$; bei dem Messwert für diesen Patienten handelt es sich allerdings um einen sehr stark von den Messwerten der übrigen Kohorte abweichenden Wert, welcher ca. doppelt so hoch wie der nächst niedrigere von 46072,2 $\mathrm{mm}^{3}$ liegt. Eine weitere, um diesen abweichenden Wert bereinigte Berechnung erbrachte einen Mittelwert von 9036,5 $\pm 8963,2 \mathrm{~mm}^{3}$ 
$(\mathrm{MW} \pm \mathrm{SD})$. Der abweichende Wert ist in die nachfolgenden Berechnungen eingeschlossen. Der für die Gesamtkohorte gemessene Minimalwert betrug 825,1 $\mathrm{mm}^{3}$. Der errechnete Median lag bei 6434,6 $\mathrm{mm}^{3}$.

Bei der durchgeführten Rangkorrelation der Läsionsvolumina mit den RNFLT-Werten nach Charles Spearman ergab sich ein Korrelationskoeffizient von -0,492 und somit ein inverser Zusammenhang. Es zeigt sich also, dass mit zunehmendem Läsionsvolumen die RNFLT abnimmt. Der errechnete naive $p$-Wert lag bei 0,0006 und damit unter dem Signifikanzniveau von $<0,001$. Dieser ist daher als hoch signifikant einzustufen. Nach Durchführung der Bonferroni-Holm-Korrektur lag der adjustierte p-Wert bei 0,0036 und damit unter dem Signifikanzniveau von $p<0,01$. Die Korrelation kann somit als sehr signifikant angesehen werden. Die nachfolgende Grafik veranschaulicht den inverssignifikanten Zusammenhang zwischen der RNFLT und dem Läsionsvolumen der Studienteilnehmer.

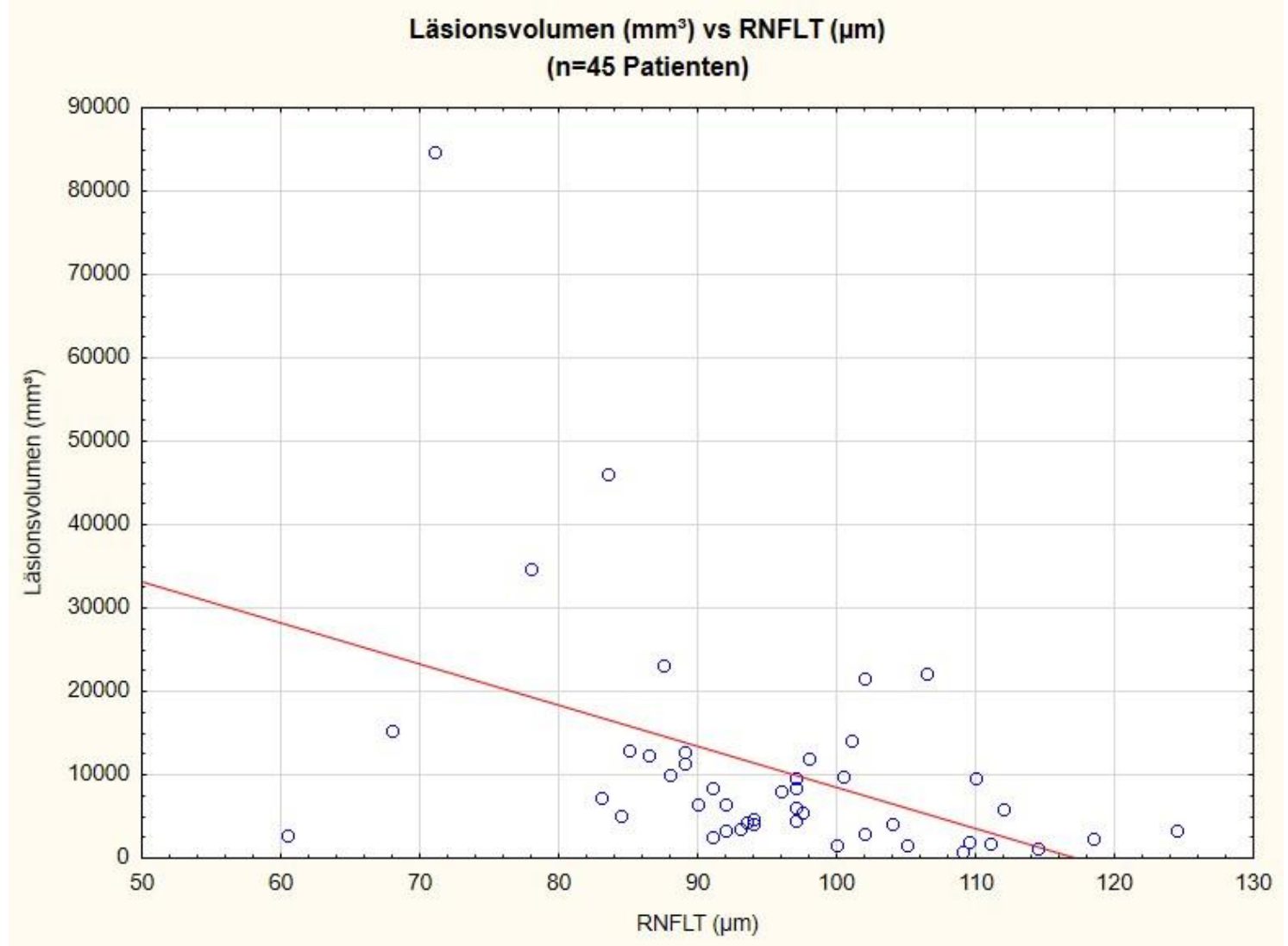

Abbildung 5: Läsionsvolumen vs. RNFLT 
Neben der Betrachtung der Läsionsherde für die Gesamtkohorte sollte eine spezielle Betrachtung der Patienten nach An- oder Abwesenheit von in den Sehstrahlungen der jeweiligen Großhirnhemisphären vorkommenden Läsionsherden erfolgen. Zusätzlich sollte eine Betrachtung der Kohorte nach der An-bzw. Abwesenheit von Läsionsherden in den jeweiligen Kleinhirnhemisphären erfolgen. Diese geplanten separaten Einteilungen der Kohorte nach Läsionsverteilung in Groß- sowie in Kleinhirnhemisphären kamen aufgrund des zu homogenen Verteilungsmusters der Läsionsherde in diesen Bereichen nicht zustande. Die Sehstrahlungen waren bei lediglich einem Patienten beidseits nicht betroffen; bei 3 Patienten war nur eine Sehbahn betroffen. Bei den übrigen 41 Patienten lagen Läsionen in den Sehstrahlungen beider Großhirnhemisphären vor. Das Verhältnis von An- zu Abwesenheit in Bezug auf Läsionen in den betrachteten Großhirnarealen lag somit bei 44:1.

Bezüglich der Kleinhirnhemisphären kamen bei 11 Studienteilnehmern keine Läsionen in diesem Bereich vor. Läsionen in nur einer Kleinhirnhemisphäre fanden sich bei 14 Patienten. Bei den 20 übrigen Patienten lagen Läsionsherde in beiden Kleinhirnhemisphären vor. Bei Betrachtung des Cerebellums lag das An- zu Abwesenheitsverhältnis der Läsionsherde daher lediglich bei 34:11.

Somit kamen bei 97,8\% der Patienten entsprechende Läsionen in mindestens einer Sehbahn und bei $75,6 \%$ der Patienten Läsionen in mindestens einer Kleinhirnhemisphäre vor.

\subsubsection{Läsionssumme}

Die Mithilfe der unter 2.4 beschriebenen Bildanalysesoftware MIPAV objektivierte Summe von Einzelläsionen in Cerebrum und Cerebellum der individuellen Patienten sollte Aufschluss über das Verteilungsmuster der Läsionsherde geben. Es stellte sich heraus, dass die Läsionen ubiquitär in der weißen Substanz nahezu aller Groß- und Kleinhirnareale vorkamen, wobei sich eine antizipierte Konzentration der Läsionen auf direkt periventrikulär gelegene Lokalisationen bestätigte. Die Ergebnisse der Zählung der Einzelläsionen werden nachfolgend dokumentiert. Bei Betrachtung der 45 
Studienteilnehmer zeigte sich eine durchschnittliche Gesamtzahl von 42,1 $\pm 33,9$ $(\mathrm{MW} \pm \mathrm{SD})$ Einzelläsionen pro Kopf. Die größte gemessene Läsionssumme ergab einen Wert 145, der erfasste Minimalwert betrug 5 Läsionsherde. Der hierbei errechnete Median lag bei 32 Läsionen.

Bei der durchgeführten Rangkorrelation der Läsionssummen mit den RNFLT-Werten nach Charles Spearman ergab sich ein Korrelationskoeffizient von -0,344 und somit ein inverser Zusammenhang. Der errechnete naive p-Wert lag bei 0,020742 und damit unter dem Signifikanzniveau von $<0,05$. Daher ist dieser als signifikant einzustufen. Nach Durchführung der Bonferroni-Holm-Korrektur lag der adjustierte $p$-Wert bei 0,041484 und somit noch immer unter dem Signifikanzniveau von $p<0,05$. Die Korrelation kann folglich als signifikant eingestuft werden. Die nachfolgende Grafik veranschaulicht den invers-signifikanten Zusammenhang zwischen der RNFLT und den Läsionssummen der Studienteilnehmer.

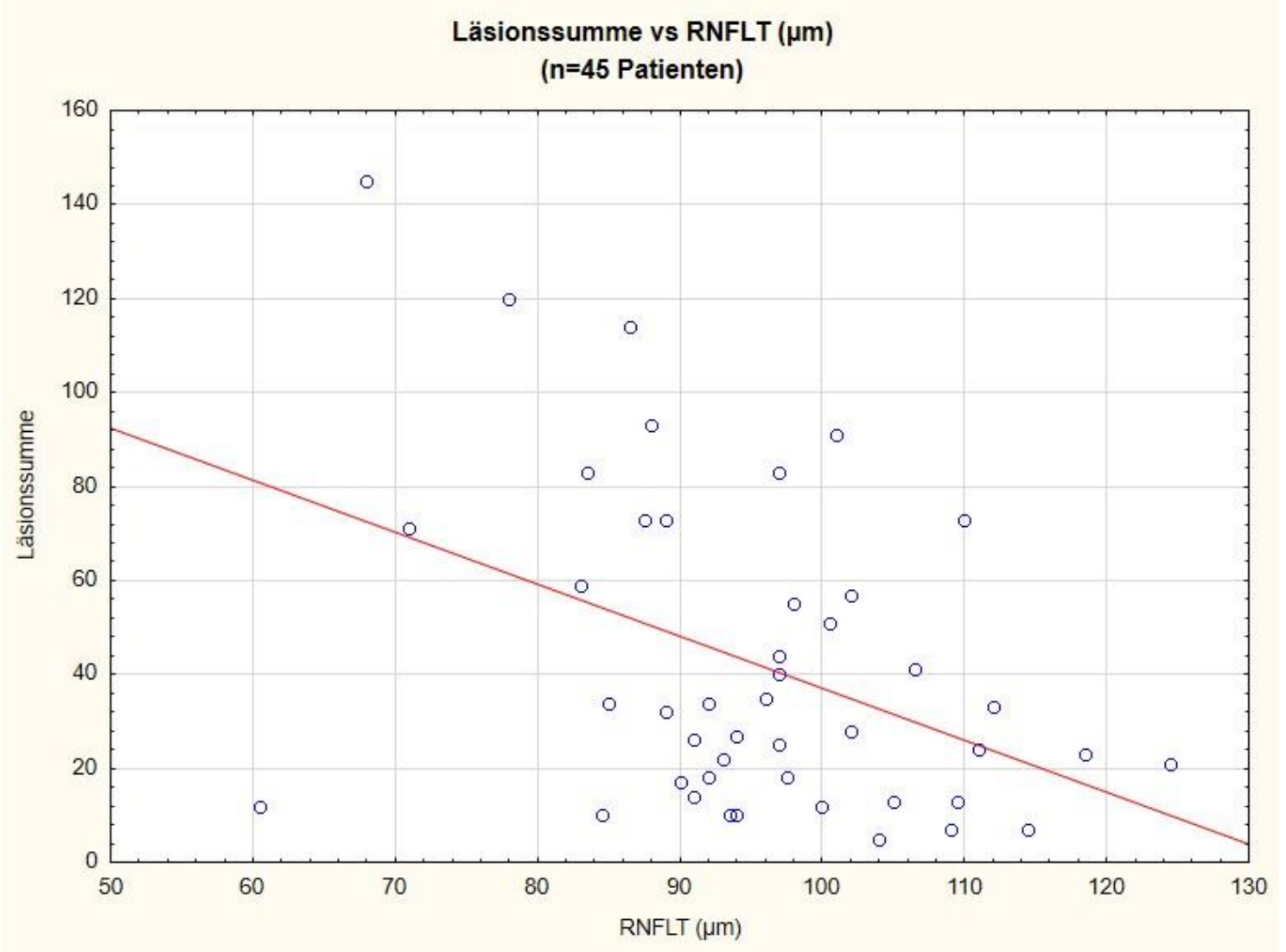

Abbildung 6: Läsionssumme vs. RNFLT 


\subsection{Ergebnisse hinsichtlich klinischer Parameter}

\subsubsection{EDSS}

Im Folgenden werden die Ergebnisse der Erhebung des Expanded Disability Status Scale (EDSS), einem Punktesystem, welches eine Aussage über die Schwere des klinischen Krankheitsverlaufs bei Multipler Sklerose erlaubt, dargelegt. Bei dem EDSS handelt es sich, anders als bei den anderen erhobenen Werten, nicht um einen numerischen, sondern um einen Punktwert. Die Bestimmung eines Mittelwerts für die Punktwerte der betrachteten Patienten ist somit nicht zielführend. Daher gibt der Median für die Betrachtung des EDSS Auskunft über die Verteilung der Punktwerte unter den Studienteilnehmern. Der hierbei bestimmte Median lag bei 0. Der maximal gemessene EDSS lag bei einem Punktwert von 3,5. Im Minimum wurde ein EDSS mit einem Wert von 0 bestimmt.

Bei der durchgeführten Rangkorrelation des EDSS mit den RNFLT-Werten nach Charles Spearman ergab sich ein Korrelationskoeffizient von -0,347 und somit ein inverser Zusammenhang. Der errechnete naive $p$-Wert lag bei 0,019385 und damit unter dem Signifikanzniveau von $<0,05$ und ist daher als signifikant einzustufen. Nach Durchführung der Bonferroni-Holm-Korrektur lag der adjustierte $p$-Wert bei 0,058155 und damit leicht über dem Signifikanzniveau von $p<0,05$. Die Korrelation ist nach der Korrektur somit nicht mehr als signifikant einzustufen. Dennoch ist eine klare Tendenz, die einen inversen Zusammenhang unterstellt, erkennbar. Die nachfolgende Grafik veranschaulicht diesen inversen Zusammenhang zwischen der RNFLT und dem EDSS der Studienteilnehmer. 


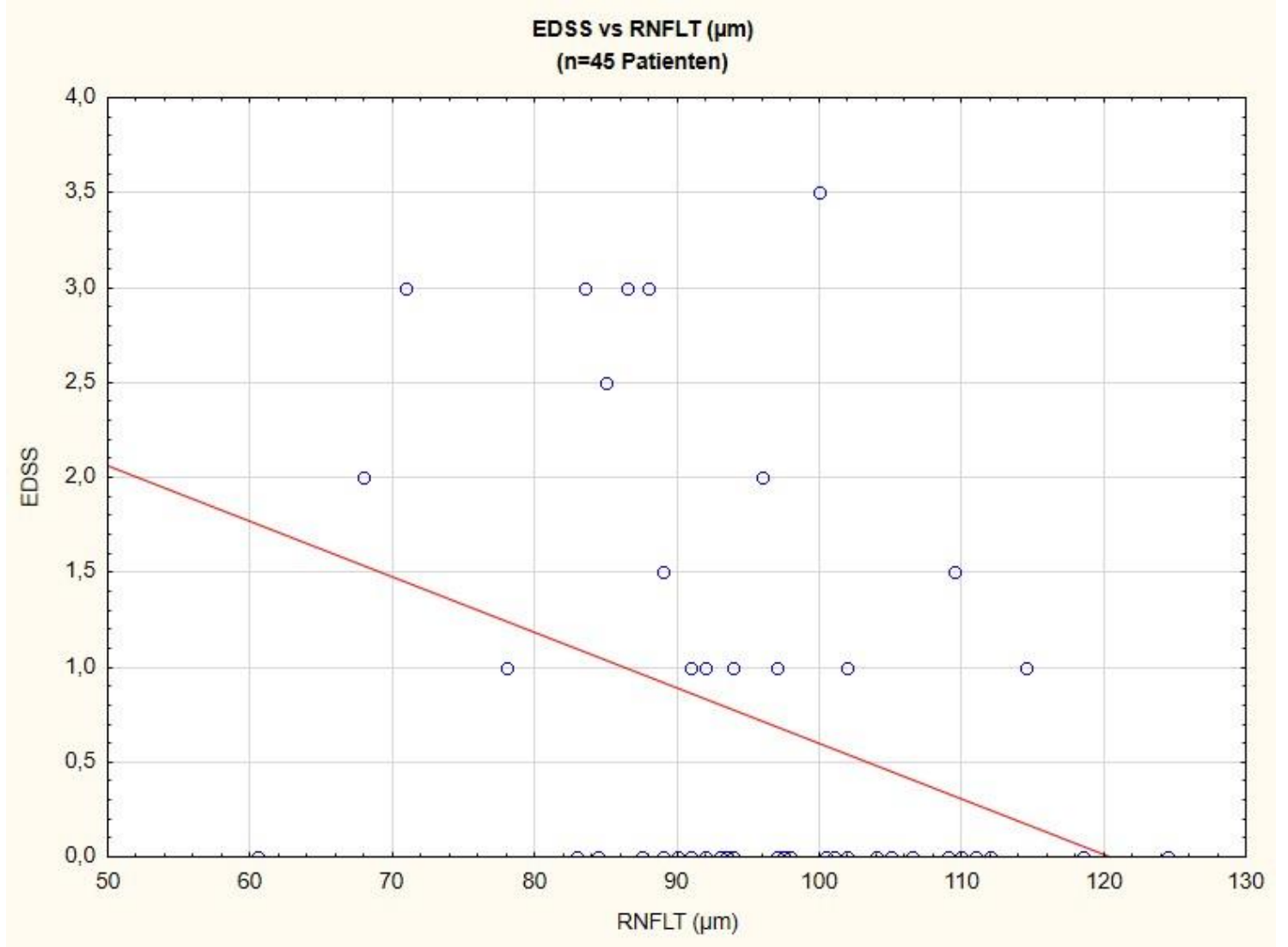

Abbildung 7: EDSS vs. RNFLT

\subsubsection{Schubsumme}

In diesem Kapitel werden die individuellen Summen der durchgemachten MS-Schübe für alle 45 Studienteilnehmer dargelegt. Die Dokumentation der Erkenntnisse wird formal den vorausgegangenen Kapiteln des Ergebnisteils folgen. Für die 45 Studienteilnehmer konnte eine durchschnittliche Gesamtzahl an durchgemachten MSSchüben von 3,1 $\pm 2(M W \pm S D)$ errechnet werden. Die maximal bestimmte Summe an MS-Schüben lag hier bei 10; die errechnete Minimalanzahl betrug einen Schub. Der ermittelte Median lag hier bei 3 Schüben.

Bei der durchgeführten Rangkorrelation der Läsionssummen mit den RNFLT-Werten nach Charles Spearman ergab sich ein Korrelationskoeffizient von -0,424 und somit ein inverser Zusammenhang. Der errechnete naive p-Wert lag bei 0,003034 und damit sowohl unter dem Signifikanzniveau von $<0,05$ als auch unter dem von $<0,01$ und ist damit als sehr signifikant einzustufen. Nach Durchführung der Bonferroni-Holm- 
Korrektur lag der adjustierte $p$-Wert bei 0,01517 und damit unter dem Signifikanzniveau von $p<0,05$. Die Korrelation kann somit als signifikant eingestuft werden. Die nachfolgende Grafik veranschaulicht den invers-signifikanten Zusammenhang zwischen der RNFLT und der Schubsumme der Studienteilnehmer.

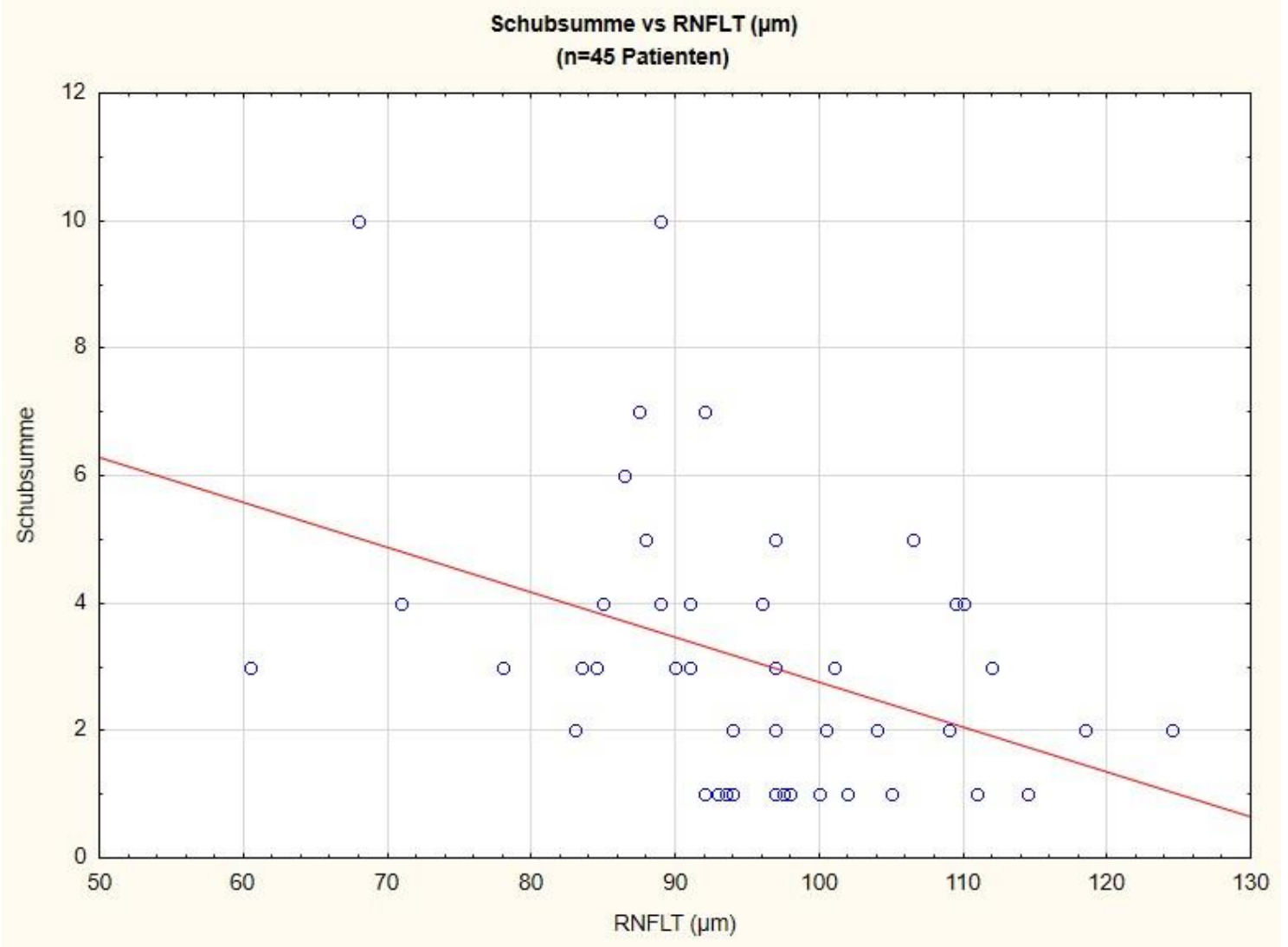

Abbildung 8: Schubsumme vs. RNFLT

\subsubsection{Krankheitsdauer}

In diesem Kapitel wird die Krankheitsdauer, welche in Monaten angegeben wird, für die 45 Studienteilnehmer betrachtet. Für die 45 Studienteilnehmer umfassende Gesamtkohorte konnte eine durchschnittliche Krankheitsdauer von 31,6 $\pm 23,6$ Monaten $(\mathrm{MW} \pm \mathrm{SD})$ errechnet werden. Die maximal bestimmte Krankheitsdauer lag hier bei 140 Monaten. Die bestimmte Minimalanzahl lag in einem Fall bei unter einem Monat (12 Tagen), da nur ganze Monate in die Betrachtung der Krankheitsdauer einflossen wird der Minimalwert bei 0 Monaten festgesetzt. Der errechnete Median lag hier bei 28 Monaten. 
Bei der durchgeführten Rangkorrelation der Krankheitsdauer mit den RNFLT-Werten nach Charles Spearman ergab sich ein Korrelationskoeffizient von -0,387 und somit ein inverser Zusammenhang. Der errechnete naive $p$-Wert lag bei 0,008712 und damit unter dem Signifikanzniveau von $<0,01$. Dieser ist damit als sehr signifikant einzustufen. Nach Durchführung der Bonferroni-Holm-Korrektur lag der adjustierte pWert bei 0,034848 und damit unter dem Signifikanzniveau von $p<0,05$. Die Korrelation kann somit als signifikant eingestuft werden. Die nachfolgende Grafik veranschaulicht den invers-signifikanten Zusammenhang zwischen der RNFLT und der Krankheitsdauer der Studienteilnehmer.

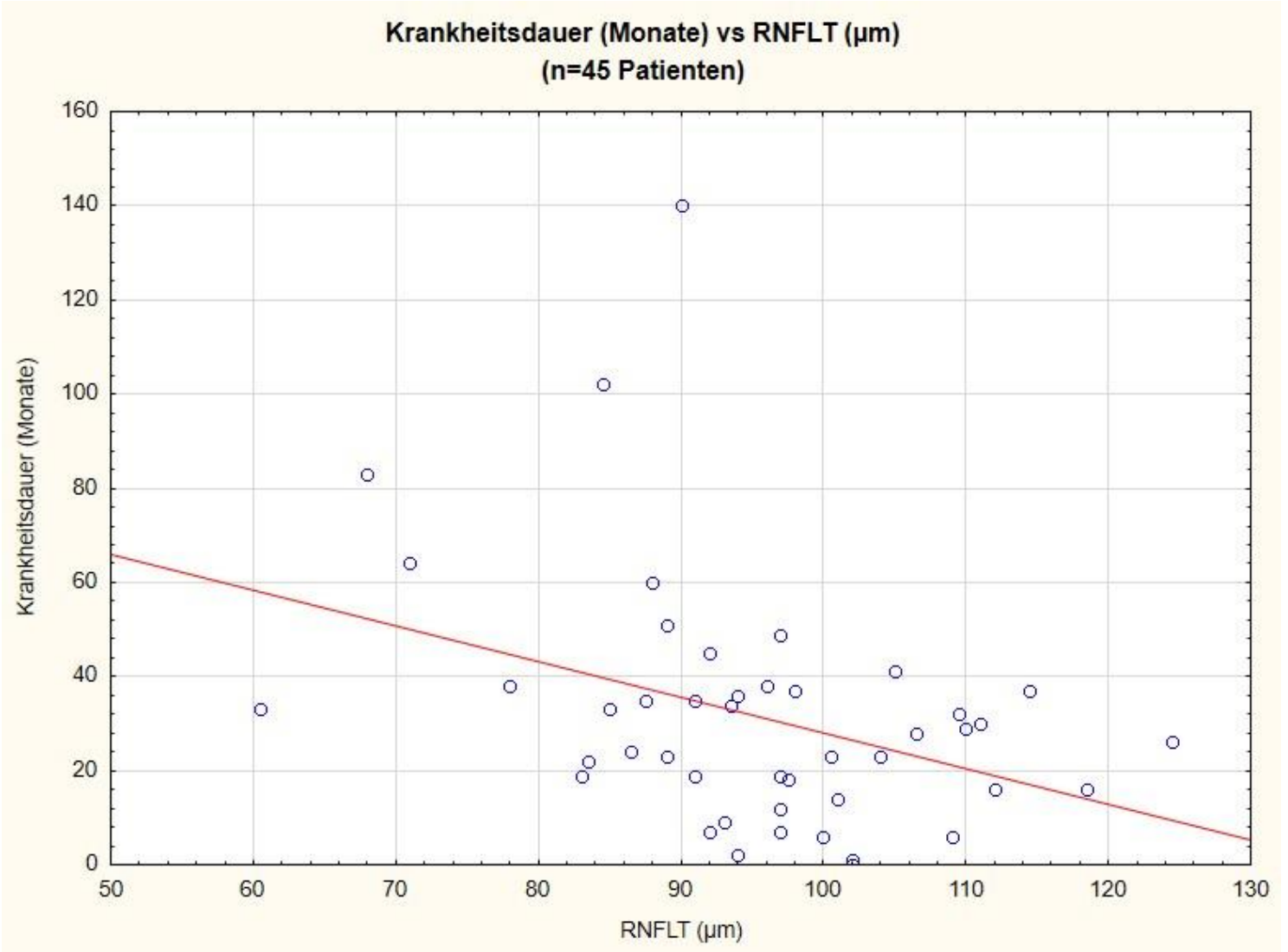

Abbildung 9: Krankheitsdauer vs. RNFLT

\subsection{Alter}

In diesem Kapitel wird das Alter der Studienteilnehmer, welches in Jahren angegeben wird, betrachtet. Für die 45 Studienteilnehmer umfassende Gesamtkohorte konnte ein durchschnittliches Alter von $16,33 \pm 1,91$ Jahren $(M W \pm S D)$ errechnet werden. Das 
maximal bestimmte Alter lag hier in einem Fall bei 19 Jahren, die Krankheitsdauer betrug in diesem Fall allerdings 19 Monate, sodass der Erkrankungsbeginn näher am oben genannten Durchschnittsalter liegt. Das bestimmte Minimalalter lag in einem Fall bei 9,75 Jahren. Der errechnete Median lag bei 16,75 Jahren.

Bei der durchgeführten Rangkorrelation der Läsionssummen mit den RNFLT-Werten nach Charles Spearman ergab sich ein Korrelationskoeffizient von -0,097 und somit ein inverser Zusammenhang. Der errechnete naive p-Wert lag bei 0,525311 und damit über dem Signifikanzniveau von $<0,05$ ist damit als nicht-signifikant einzustufen. Da dieser $\mathrm{p}$-Wert der größte der Berechneten war, lag der adjustierte $p$-Wert nach Durchführung der Bonferroni-Holm-Korrektur ebenfalls bei 0,525311. Die nachfolgende Grafik veranschaulicht den nicht-signifikanten Zusammenhang zwischen der RNFLT und dem Alter der Studienteilnehmer. Man beachte hierbei die relativ horizontal durch die Punkte des Streudiagramms verlaufende Gerade. Verglichen mit den Geraden der zuvor aufgeführten Streudiagramme für die signifikanten Korrelationen, fällt die Mittlinie dieses Diagramms bei zunehmender RNFLT nur diskret ab.

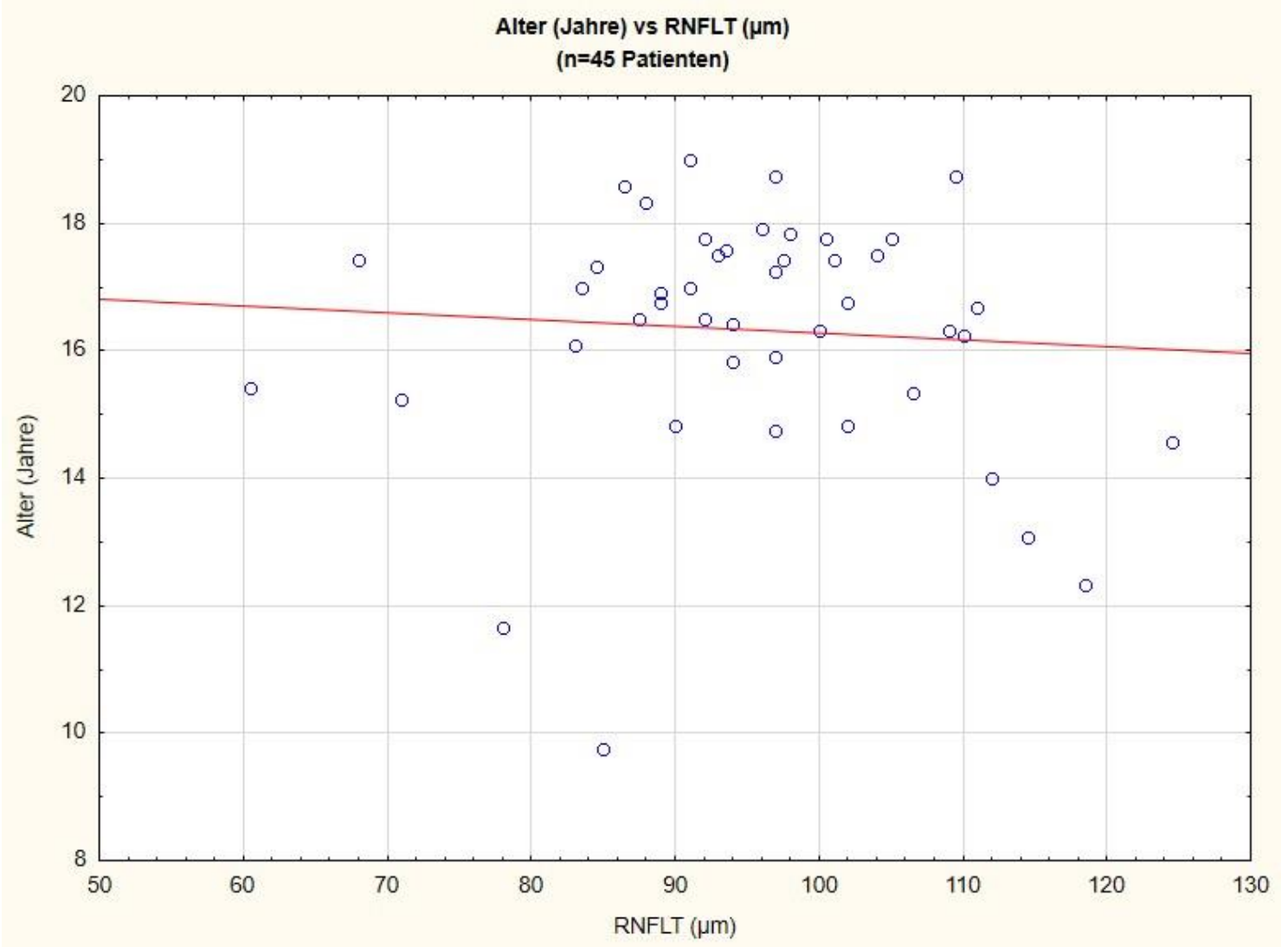

Abbildung 10: Alter vs. RNFLT 


\section{Diskussion}

Zielsetzung dieser Arbeit war es zu untersuchen, ob die Ergebnisse der für adulte MSPatienten bereits gefundenen Zusammenhänge zwischen der Abnahme der mittels OCT erhobenen RNFLT und der Zunahme der mittels MRT bestimmten Läsionslast (Scheel et al. 2014; Sinnecker et al. 2015) auch für pädiatrische MS-Patienten reproduzierbar sind. Die hierbei ermittelten Ergebnisse sollen im Folgenden diskutiert und in den Kontext der Fachliteratur eingeordnet werden.

Wie in Kapitel 3 aufgeführt, konnte ein statistisch signifikanter, korrelativer Zusammenhang zwischen der Zunahme der individuellen Läsionsvolumina der Läsionsherde und der gleichzeitigen Abnahme der retinalen Nervenfaserschichtdicke nachgewiesen werden. Der Nachweis für die signifikante inverse Korrelation zwischen den hier untersuchten klinischen Parametern und der RNFLT konnte, mit der Ausnahme der nicht-signifikanten Korrelation zwischen der RNFLT und dem Patientenalter, erbracht werden. Dieser singuläre Befund kann allerdings der mit 45 Patienten geringen Größe bzw. der gewissen Zufallsschwankungen unterliegenden Zusammenstellung der Patientenkohorte geschuldet sein.

Als Kernaussage der Datenauswertung kann somit festgehalten werden, dass für die untersuchten pädiatrischen Patienten ein mittels MRT erhobenes MS-Läsionsvolumen sowie die erhobene Läsionssumme signifikant invers mit der mittels OCT erhobenen RNFLT korreliert. Des Weiteren bestehen signifikant inverse Korrelationen zwischen der RNFLT und den erhobenen klinischen Parametern. Einzige Ausnahme bildet die nicht-signifikante Korrelation zwischen der RNFLT und dem Patientenalter.

\section{Geschlechter- und Altersverteilung}

Bereits im Jahr 1997 konnte im Rahmen der Langzeitstudie von Jacobson et al., welche Publikationen ab dem Erscheinungsjahr 1965 berücksichtigt, aufgezeigt werden, dass Frauen im Schnitt ca. 2,7-mal häufiger an 24 zuvor definierten Autoimmunerkrankungen erkrankten als Männer (Jacobson et al. 1997). Es konnte also eine klare Mehrbelastung des weiblichen Geschlechts im Verhältnis für (24) Autoimmunerkrankungen im Allgemeinen dokumentiert werden (Jacobson et al. 
1997). Im Rahmen der Studie von Ansar Ahmed et al. konnten stellenweise deutlich stärkere Mehrbelastungen des weiblichen Geschlechts ermittelt werden, beispielsweise bei der Hashimoto-Thyreoiditis, wo das Geschlechterverhältnis bei $\mathrm{F}$ : M = 25-50: 1 lag (Ansar Ahmed et al. 1985). In Ihrer Publikation von 2001 zeigte Whitacre auf, dass das Geschlechterverhältnis auch bei der Multiplen Sklerose im Speziellen einen Gradienten hin zum weiblichen Geschlecht aufweist (Whitacre 2001). Die relevante Fragestellung, wie es zu diesen teilweise sehr eindeutigen Erkrankungsgradienten der MS auf Seiten des weiblichen Geschlechts kommt, sollte Gegenstand längerfristig angelegter Studien sein, welche krankheitsrelevante Differenzen zwischen weiblichem und männlichem Immunsystem und Hormonhaushalt aufzeigen, aber auch individuelle Umweltfaktoren berücksichtigen.

Es stellt sich die Frage, ob die Aussagekraft dieser Studie dadurch steigt, dass das Verhältnis von weiblichen zu männlichen Studienteilnehmern in dieser aus 45 Patienten bestehenden Kohorte mit 31:14 in etwa der aktuell geschätzten, globalen Geschlechterverteilung bei der Multiplen Sklerose von $\mathrm{F}: \mathrm{M}=2: 1$ entspricht (MS Atlas 2008, Atlas of MS 2013). Obgleich diese Beobachtung dem nachfolgend näher diskutierten globalen Geschlechterverhältnis entspricht, sei darauf verwiesen, dass die Zusammenstellung der Patientenkohorte zufällig anhand der Ein- und Ausschlusskriterien und nicht dem Geschlecht nach erfolgte.

Die Definition der MS des Kindes- und Jugendalters beinhaltet laut Pohl einen Krankheitsbeginn vor dem 17. Lebensjahr und am ehesten ein metaanalytisches Geschlechterverhältnis von $\mathrm{F}: \mathrm{M}=2,3: 1$. Besondere Aufmerksamkeit verdiene hierbei die Gruppe der sogenannten „true childhood“ (Pohl 2007, Seite 13) MS-Patienten mit einem Krankheitsbeginn vor dem 11. Lebensjahr, deren Geschlechterverhältnis metaanalytisch bei $F: M=0,9: 1$ liege und damit (noch) keine Präferenz des weiblichen Geschlechts aufweise. Die Mehrbelastung des weiblichen Geschlechts entwickle sich erst im Rahmen der Pubertät. Hier käme es bei Kindern zwischen 10 und 15 Jahren zu einem Erkrankungsverhältnis von $F: M=2,6: 1$. Die größte Mehrbelastung des weiblichen Geschlechts findet sich Pohl zufolge bei Patienten im Alter zwischen 13 und 15 Jahren, hier erreiche das Geschlechterverhältnis mit $F: M=$ 3,1 : 1 sein Maximum und liege damit deutlich oberhalb des Verhältnisses bei der adulten MS mit F : M = $2: 1$ (Pohl 2007). Das Durchschnittsalter der 45 Teilnehmer 
dieser Studie entspricht mit 16,33 \pm 1,91 Jahren (MW \pm SD) weniger der "true childhood" (Pohl 2007, Seite 13) und eher der adoleszenten MS, deren Geschlechterverhältnis weitgehend dem für die adulte MS entspricht.

In Kanada als Beispiel für einen westlichen Industriestaat durchgeführte Studien an unter MS leidenden Patienten konnten die angenommene Geschlechterverteilung zunächst ebenfalls bei ca. $F: M=2: 1$ festsetzen, berichten allerdings von einer progressiven Verschiebung des Verhältnisses zu Seiten des weiblichen Geschlechts (Orton et al. 2006). Weitere Studien konnten diesen Trend reproduzieren und bestätigen (Ramagopalan et al. 2009). Obgleich der oben genannten zufälligen Kohortenzusammenstellung unterworfen, konnte die erfasste Geschlechterverteilung für die in dieser deutschen Studie untersuchten 45 pädiatrischen Patienten mit $F: M=$ 31 : 14 in etwa diesem für Kanada erfassten Trend nachfolgen. Haliloglu et al. konnten hingegen aufzeigen, dass für die 28 pädiatrischen Patienten ihrer Studie ein Geschlechterverhältnis von $\mathrm{F}: \mathrm{M}=1,5: 2 \mathrm{bzw}$. $\mathrm{F}: \mathrm{M}=0,75$ und somit eine Lage des Verteilungsgradienten auf Seiten der männlichen Studienteilnehmer vorlag (Haliloglu et al. 2002). Das von Haliloglu et al. beobachtete Geschlechterverhältnis widerspricht altersentkoppelten Inzidenzen bzw. Mehrheitsverhältnissen (MS Atlas 2008, Atlas of MS 2013, Jacobson et al. 1997) und betrifft in dieser Studie an Patienten im Alter zwischen 5 und 16 Jahren lediglich pädiatrische Patienten mit definitiver MS unter Ausschluss der nicht-definitiven Fälle. Diese Ergebnisse deckten sich mit im Rahmen weiterer Studien gewonnener Erkenntnisse zur Umkehr der Geschlechterverteilung bei early-onset-MS bzw. Multipler Sklerose im Kindesalter; zitiert wurden hier Teile der Ergebnisse von Ruggieri et al., welche für Kinder unter 24 Monaten ein Geschlechterverhältnis von $F: M=0,6: 1$ ermittelten sowie die Ergebnisse von Hanefeld et al., welche für pädiatrische Patienten ein Geschlechterverhältnis von $F: M$ = $1: 4$ bestimmen konnten (Hanefeld et al. 1991; Ruggieri et al. 1999). Ergänzend ist allerdings zu erwähnen, dass Ruggieri et al. 1999 für ihre Gesamtkohorte von 49 Patienten im Alter von unter 6 Jahren ein Geschlechterverhältnis von $F: M=1,4: 1$ ermittelten. Sie selbst hoben die Diskrepanz der gewonnenen Erkenntnisse zu denen für adulte MS-Patienten (Geschlechterverhältnis von $F: M=2: 1$ ) und zu jenen im Alter zwischen 6 und 15 Jahren (Geschlechterverhältnis von $F: M=2,2-3: 1$ ) hervor. Eine nähere Erörterung der Reproduzierbarkeit des möglicherweise zunehmenden 
Gradienten in der Geschlechterverteilung der MS in weiteren Staaten sollte, stetig aktualisiert, vorgenommen werden. Besondere Aufmerksamkeit sollte dabei dem Geschehen in den Industriestaaten sowie der Identifikation zugrundeliegender auslösender Mechanismen bzw. Umweltfaktoren zukommen. Großes Augenmerk sollte auch auf den Vergleich identifizierter Ursachen für die Multiple Sklerose in Industriestaaten und Nicht-Industriestaaten gelegt werden.

\section{OCT und Multiple Sklerose}

Der mögliche Einsatz des OCT als Screening- und Follow-up-Untersuchung für die Multiple Sklerose, insbesondere bei Kindern, sollte im Lichte der hier gewonnenen Erkenntnisse diskutiert werden.

Für die weitere und genauere Analyse der Bedeutung dieser Arbeit im Kontext der aktuellen MS-Forschung ist es erforderlich, etwaige altersspezifische Unterschiede in Erscheinung und Verlauf der Multiplen Sklerose zu diskutieren. Daher ist es notwendig, die im MRT als Läsionsherde imponierenden Nerven- bzw. Myelinscheidenatrophien bei den unterschiedlichen Verlaufsformen der MS, namentlich der sogenannten adultonset-Form sowie der early-onset-Form, zu vergleichen. Darüber hinaus sollten die Ergebnisse dieses Vergleiches, welcher im Rahmen der nachfolgend beschriebenen Studie vorgenommen wurde, unter Berücksichtigung der Erkenntnisse aus der vorliegenden Studie, betrachtet werden. In Ihrer Studie von 2009 konnten Waubant et al. aufzeigen, dass die MS-typischen Läsionsherde bei der early-onset-Form der MS im Allgemeinen zahlreicher sind als bei der adult-onset-Form (Waubant et al. 2009).

Vergleicht man nun diese MRT-basierten Ergebnisse mit denen aus OCT-gestützten Verfahren, wird ersichtlich, dass unter Betrachtung der nachfolgend genannten Studien für die OCT bezüglich des Alters inverse Verhältnisse zu gelten scheinen. Als Beleg für diese These kann der direkte Vergleich der Korrelationsergebnisse zwischen der Abnahme der RNFLT und der gleichzeitigen Zunahme der mittels MRT ermittelten Läsionslast in dieser Studie mit den Ergebnissen der Studie von Sinnecker et al. von 2015 herangezogen werden. Während Sinnecker et al. in ihrer an adulten Patienten durchgeführten Studie einen inversen Zusammenhang zwischen dem vermehrten Auftreten von Sehbahnläsionen und der gleichzeitigen Minderung der retinalen 
Nervenfaserschichtdicke aufzeigen konnten, wurden in dieser Studie ähnliche Ergebnisse für pädiatrische Patienten reproduziert. Allerdings scheint die OCTUntersuchung für adulte MS-Patienten stärkere Verringerungen der RNFLT zu ermitteln. Während Sinnecker et al. bei 19 Patienten ohne NNO in einem Alter von durchschnittlich $34 \pm 9$ Jahren (MW \pm SD) eine durchschnittliche RNFLT von $89 \pm 13 \mu \mathrm{m}$ (MW \pm SD) ermitteln konnten (Sinnecker et al. 2015), wurde für die 45 pädiatrischen Patienten dieser Studie eine geringere Minderung der retinalen Nervenfaserschichtdicken von 95,41 $\pm 12,71 \mu \mathrm{m}(\mathrm{MW} \pm \mathrm{SD}$ ) errechnet. Eine mögliche Erklärung für das Zustandekommen dieser Differenz ist der in der Publikation von Sinnecker et al. mit 19 Patienten, verglichen mit den 45 Patienten dieser Studie, geringere Anteil an betrachteten Studienteilnehmern. Auch der Einfluss der von Alasil et al. beschriebenen altersabhängigen Atrophie der RNFLT, auf welche im Verlauf noch eingegangen wird, ist als mögliche Teilursache zu berücksichtigen (Alasil et al. 2013).

Unter Annahme der Repräsentativität dieser Beobachtung wäre der Schluss zulässig, dass die Einsatzmöglichkeiten der Optischen Kohärenztomographie in Diagnostik und Verlaufsbeobachtung der MS bei adulten Patienten erfolgversprechender scheinen als bei pädiatrischen Patienten, da bei den Erstgenannten mit einer stärkeren Atrophie der RNFLT zu rechnen wäre.

Betrachtet man die Studie von Waubant et al., die besagt, dass bei der early-onsetForm der MS im Durchschnitt eine größere Läsionssumme ermittelt werden konnte als bei der adult-onset-Form (Waubant et al. 2009), wird unter Berücksichtigung der durchschnittlichen RNFLT dieser Studie mit der aus der Studie von Sinnecker et al. ersichtlich, dass zwischen der Läsionssumme und der ermittelten RNFLT kein uneingeschränkt inverser Zusammenhang für alle Altersgruppen bestehen muss. Der in dieser Studie gefundene inverse Zusammenhang zwischen RNFLT und Läsionssummen bei pädiatrischen Patienten verdient, in Zusammenschau mit den Ergebnissen für adulte Patienten von Waubant et al. (weniger Läsionen bei der adult-onset-MS) und Sinnecker et al. (geringere RNFLT bei den untersuchten adulten Patienten im Vergleich zu den pädiatrischen Patienten dieser Studie) daher besondere Aufmerksamkeit (Waubant et al. 2009; Sinnecker et al. 2015).

Die Zusammenschau suggeriert, dass bei adulten Patienten ein, verglichen mit pädiatrischen Patienten, schwächer inverser, oder gar linearer Zusammenhang 
(weniger Läsionen, geringere RNFLT) bestehen könnte. Ein solcher Zusammenhang stünde dem signifikant-inversen bei pädiatrischen Patienten gegenüber. Dieser mögliche Widerspruch bestünde allerdings vorbehaltlich einer Läsionssumme, welche sich, anders als in der Studie von Sinnecker et al., nicht-signifikant zur Abnahme der RNFLT verhält. Bei pädiatrischen Patienten wäre daher mit stärker signifikanten inversen Zusammenhängen und damit mit einer zuverlässigeren diagnostischen Aussagekraft des Verhältnisses von MRT und OCT zu rechnen. Beim Vergleich dieser drei Studien gilt es allerdings zu beachten, dass die zur Diagnostik angewandten Verfahren, im speziellen MRT und OCT, nicht nach einheitlichen Maßstäben erfolgten und dies bei der Einstufung der Aussagekraft dieser Beobachtung einschränkend zu berücksichtigen ist.

In Ihrer Studie an 31 an einem klinisch isolierten Syndrom leidenden Patienten konnten Filippi et al. aufzeigen, dass weitreichende axonale Schädigungen bereits als Frühzeichen der Multiplen Sklerose noch vor Auftreten der Sichtbarkeit im MRT nachweisbar sind (Filippi et al. 2003). Der Nachweis der Pathologie erfolgte über die Quantifizierung der sogenannten whole-brain N-acetylaspartate (WBNAA). Filippi et al. belegen in ihrer Studie daher die Existenz einer klaren Latenz vom Krankheitsbeginn der MS bzw. deren Nachweisbarkeit über andere diagnostische Mittel bis zur Nachweisbarkeit mit der als Standard anerkannten Untersuchungsmethode mittels MRT. Es drängt sich daher erneut die Frage auf, ob das in dieser Studie erfolgreich zum Nachweis von pathologischen Veränderungen der RNFLT eingesetzte OCT in dieser Latenz- und Frühphase der Erkrankung zum Nachweis möglicherweise bereits aufgetretener, pathologischer Veränderungen der RNFLT geeignet ist und welche Rückschlüsse derartige Veränderungen auf die Krankheitsaktivität der Patienten zuließen. Unter Berücksichtigung der Ergebnisse von Filippi et al. steht auch zur Prüfung, inwieweit sich die OCT als ergänzende diagnostische oder mögliche Screening-Methode eignet, um zu klären, ob bereits bei Auftreten von ersten Frühsymptomen die Durchführung einer OCT-Untersuchung gerechtfertigt ist und eine Abnahme der RNFLT beobachtet werden kann.

Ähnlich wie in dieser Studie konnten Yeh et al. in ihrer Studie aus dem Jahr 2014 nachweisen, dass bei einer 37 Patienten starken Gruppe pädiatrischer MS-Patienten, im Vergleich mit einer gesunden, 18 Studienteilnehmer umfassenden pädiatrischen 
Kontrollgruppe, eine deutliche Abnahme der retinalen Nervenfaserschichtdicke zu beobachten war (Yeh et al. 2014). Die Erhebung der RNFLT erfolgte unter Zuhilfenahme eines CIRRUS OCT von CARL ZEISS MEDITEC. Nach Yeh et al. betrug die Reduktion der RNFLT bei den erkrankten Patienten im Vergleich zur RNFLT der Kontrollgruppe einen prozentualen Anteil von 25,6\%. Diese vielversprechende Differenz kann als Beleg für die Relevanz des OCT in der Diagnostik der Multiplen Sklerose herangezogen werden, insbesondere unter Berücksichtigung der in der vorliegenden Studie aufgezeigten, inversen Korrelation zwischen der Zunahme der mittels MRT bestimmten Läsionsvolumen und der damit einhergehenden, mittels OCTUntersuchungen bestimmten, Atrophie der retinalen Nervenfaserschichtdicke. Die Aussage der Studie von Yeh et al. wird durch die Ergebnisse dieser Studie daher weitgehend bestätigt.

Die Zusammenschau der Ergebnisse sowohl dieser beiden als auch der vorangegangenen Studien und Publikationen kann, wenn nicht als Beweis, so doch zumindest als klarer Hinweis für die wachsende Relevanz des OCT als sinnvolle Ergänzung des MRT in Diagnostik und Verlaufsbeurteilung der Multiplen Sklerose bei Kindern und Erwachsenen herangezogen werden.

Bezüglich des u. a. optische Defizite erfassenden EDSS konnten Abalo-Lojo et al. im Rahmen einer Studie an 88 MS-Patienten in einem Alter von durchschnittlich 39,19 \pm 9,62 (MW $\pm S D)$ eine invers-signifikante Korrelation $(P=0,003)$ zur RNFLT zeigen (AbaloLojo et al. 2014). Für die 45 Teilnehmer dieser Studie ohne NNO-Anamnese konnte mit einem nicht-adjustierten $p$ von gerundet 0,02 somit ebenfalls eine signifikante Korrelation für pädiatrische Patienten ermittelt werden. Da die oben genannte Studie allerdings Patienten mit NNO-Anamnese einschloss und damit nicht den ausschließlich MS-bedingten Einfluss auf die RNFLT misst, wäre bei Ausschluss besagter Patienten von einer Abschwächung der Korrelation auszugehen. Die hier ermittelten Korrelationen zwischen MS-bedingter Minderung der RNFLT und mittels EDSS erfasster Klinik sind durch den Ausschluss von NNO-Patienten von dem damit einhergehenden Einfluss auf die RNFLT befreit und somit eher als repräsentativ einzustufen.

Betrachtet man die nachfolgenden Publikationen hinsichtlich der Altersabhängigkeit der RNFLT bei gesunden Studienteilnehmern, welche beispielsweise als Mitglieder von 
Kontrollgruppen betrachtet wurden, fällt die im Verhältnis zu MS-Patienten kräftige RNFLT auf.

Während bei der Studie von Sinnecker et al. die RNFLT bei 19 nNNO-MS-Patienten in einem Alter zwischen 19 und 49 Jahren (MW $\pm S D: 34 \pm 9$ ) durchschnittlich bei $89 \pm 13$ $\mu \mathrm{m}$ lag, konnte für die 12 gesunden Kontrollen in einem Alter von 20 bis 54 Jahren eine durchschnittliche RNFLT von $98 \pm 9 \mu \mathrm{m}$ ermittelt werden. Das Durchschnittsalter der Kontrollen lag bei $34 \pm 11$ Jahren. Noch größer war die Differenz zu den 11 untersuchten MS-Patienten mit NNO-Anamnese in einem Alter von 29 bis 46 Jahren

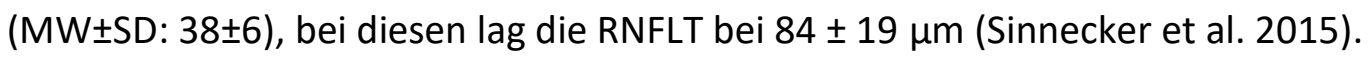

Für die nNNO-Augen der in dieser Studie betrachteten 45 MS-Patienten in einem Alter zwischen 9,75 und 19 Jahren (MW $\pm S D$ : 16,33 \pm 1,91) konnte eine durchschnittliche RNFLT von 95,41 \pm 12,71 $\mu \mathrm{m}$ (bzw. für die separate Betrachtung der 74 nNNO-Augen: $94,79 \pm 14,2 \mu \mathrm{m})$ bestimmt werden. Obgleich für diese Studie keine Kontrollgruppe vorliegt, können die nachfolgend näher betrachteten Studien von Salchow et al. und Alasil et al. beim Ermessen von RNFLT-Referenzwerten für gesunde Kinder und Jugendliche herangezogen werden.

Für die 92 Teilnehmer der Studie von Salchow et al. in einem Alter zwischen 4 und 17 Jahren (MW \pm SD: 9,7 $\pm 2,7$ ) konnte eine durchschnittliche RNFLT von $107 \pm 11,1 \mu \mathrm{m}$ ermittelt werden (Salchow et al. 2006). Aufgrund des, verglichen mit den Teilnehmern dieser Studie, geringeren Durchschnittsalters kann die Kohorte von Salchow et al. nicht als alleinige Refrenzgruppe angesehen werden. Daher sind die Erkenntnisse von Alasil et al. für die Eingrenzung der RNFLT-Werte bei Gesunden ebenfalls zu betrachten. Alasil et al. konnten für die von ihnen untersuchten 190 gesunden Kinder und Erwachsenen in einem Alter zwischen 9 und 86 Jahren (MW $\pm S D$ : 53,7 \pm 16,3) durchschnittliche RNFLT-Werte von 97,3 \pm 9,6 $\mu \mathrm{m}$ bestimmen. Hier sei allerdings auf das hohe Durchschnittsalter verwiesen, welches die ermittelte RNFLT eher mit den adulten Kontrollen aus der Studie von Sinnecker et al., mit Werten von $98 \pm 9 \mu \mathrm{m}$, vergleichbar macht (Alasil et al. 2013; Sinnecker et al. 2015). Wesentlich ist allerdings, dass Alasil et al. neben den genannten Erkenntnissen einen altersabhängigen Trend identifizieren konnten: Mit jeder Lebensdekade verringert sich die RNFLT um ca. 1,5 $\mu \mathrm{m}$. 
Korrigiert man nun den ermittelten RNFLT-Durchschnitt von 97,3 $\mu \mathrm{m}$ für die Patienten in einem Alter von durchschnittlich 53,7 Jahren auf das Durchschnittsalter der Patienten dieser Studie von 16,33 Jahren, so ergibt sich aufgrund des ca. 37,4 Jahre umfassenden Altersunterschieds eine RNFLT-Differenz von ca. 5,6 $\mu \mathrm{m}$. Die RNFLT läge auf Grundlage dieser Werte bei ca. 102,9 $\mu \mathrm{m}$ und damit für Gesunde ca. 7,5 $\mu \mathrm{m}$ höher als der Durchschnitt der 45 Teilnehmer dieser Studie (MW \pm SD: 95,41 $\pm 12,71 \mu \mathrm{m}$ ) bzw. bei separater Betrachtung der 74 nNNO-Augen (MW \pm SD: $94,79 \pm 14,2 \mu \mathrm{m}$ ) bei ca. 8,1 $\mu \mathrm{m}$. Diese Werte sind allerdings aufgrund des hohen Durchschnittsalters und der hohen Standardabweichung für die Teilnehmer der Studie von Alasil et al. einschränkend zu betrachten. Ein Vergleich mit den Teilnehmern der Studie von Salchow et al., deren Durchschnittsalter und zugehörige Standardabweichung eine geringe Differenz zu den Werten der Teilnehmer dieser Studie aufweisen, wird nachfolgend vorgenommen.

Bei Betrachtung der RNFLT-Werte dieser Studie und denen aus der Studie von Salchow et al. (MW \pm SD: $107 \pm 11,1 \mu \mathrm{m}$ ) kann, unter Berücksichtigung des vorbeschriebenen Trends, aufgrund des geringen durchschnittlichen Altersunterschieds von einer RNFLTDifferenz von über $10 \mu \mathrm{m}$ (ca. 10,6 $\mu \mathrm{m}$ ) ausgegangen werden. Obgleich auch an der UMG für pädiatrische Patienten noch keine RNFLT-Grenzwerte festgelegt wurden, kann, wenn man den nach Alasil et al. korrigierten Referenzbereich von Salchow et al. als normwertig zugrunde legt, von einer starken Minderung der RNFLT bei pädiatrischen MS-Patienten ausgegangen werden. Das OCT wäre daher im Falle dieser Studie geeignet gewesen, eine ca. $10 \mu \mathrm{m}$ umfassende Differenz zwischen den mittleren RNFLT-Werten von nNNO-MS-Patienten und Gesunden zu erfassen. Die Validität dieser Annahme setzt allerdings den Ausschluss von Augen mit NNO-Anamnese voraus, da bei diesen eine stärkere Minderung der RNFLT unterstellt werden muss. Dieser Zusammenhang konnte sowohl für die von dieser Studie ausgeschlossenen NNO-Augen mit RNFLT-Werten von 84,43 $\pm 15,03 \mu \mathrm{m}$ als auch für die aus der Studie von Sinnecker et al. mit Werten von $84 \pm 19 \mu \mathrm{m}$ reproduziert werden. Da es sich bei den 45 Teilnehmern dieser Studie allerdings um MS-Patienten ohne NNO-Anamnese handelt, kann bei den vorliegenden RNFLT-Minderungen von einem mittels OCT erfassten, weitgehend separaten Einfluss der MS auf die Atrophie der retinalen Nervenfaserschichtdicke ausgegangen werden. 
MRT und MS-Diagnostik

Die zentrale Rolle der Magnetresonanztomographie in der modernen Diagnostik und Erforschung der Multiplen Sklerose soll nachfolgend diskutiert werden.

Bei der Einordnung dieser Studie in die Fachliteratur sind mögliche, im Rahmen anderer Studien bereits entdeckte Fehlerquellen zu berücksichtigen. So konnte beispielsweise gezeigt werden, dass bei der MRT-gestützten Untersuchung von MSLäsionsherden in einem im 1,5 Tesla MRT als konfluierend imponierendem Läsionsherd unter Zuhilfenahme eines 4 Tesla MRT häufig mehrere Einzelläsionen identifiziert werden können (Davis 2014). Davis schlussfolgert, dass dieser Umstand auch für die Segmentation großer, konfluierender periventrikulärer Läsionen, die mittels konventionellem MRT dargestellt werden, problematisch sein könnte. Diese Erkenntnis ist bei der Sichtung der im Rahmen dieser Studie gewonnenen Daten berücksichtigt worden. Es kann geschlussfolgert werden, dass nach Davis eine MR-Bildgebung mit einer Feldstärke von 1,5 Tesla eine mögliche Fehlerquelle bei der Bestimmung individueller Läsionssummen und -volumina von MS-Patienten darstellt. Im Rahmen dieser Studie kam für die Erhebung der zuvor genannten Parameter allerdings ein Magnetresonanztomograph mit einer Feldstärke 3 Tesla zum Einsatz. Die hier verwendete, im Vergleich zur von Davis beschriebenen konventionellen MRT mit lediglich 1,5 Tesla, doppelt so hohe Feldstärke im SIEMENS TRIMTRIO-Gerät lässt auf geringere Genauigkeitseinbußen schließen.

Wie bereits in der Publikation von Yulin Ge dargelegt wurde, ist die sogenannte Normal Appearing White Matter, kurz NAWM, maßgeblich an der gesamten Läsionslast beteiligt, wird von aktueller MRT-Technologie allerdings nicht als pathologisch identifiziert (Ge 2006). Da die hier vorliegende Studie eine klare Korrelation zwischen der Reduktion der retinalen Nervenfaserschichtdicke und dem Auftreten klinisch messbarer Begleiterscheinungen bzw. Symptome bei pädiatrischen Patienten nachweist, ist die Frage zu stellen, ob die durch NAWM verursachte diagnostische Lücke durch eine Optimierung und Weiterentwicklung der OCT teilweise zu schließen ist. Bereits in ihrer Publikation aus dem Jahr 2002 kamen O'Connor und die Canadian Multiple Sclerosis Working Group zu dem Schluss, dass lediglich eine schwache Korrelation zwischen klinischer und MRT-gestützter Messbarkeit der Krankheitsaktivität bei Multipler Sklerose vorliegt ( $O^{\prime}$ Connor und Canadian Multiple 
Sclerosis Working Group 2002). Zu einer ähnlichen Feststellung gelangten auch Banwell und Sled. Diese bescheinigen in ihrer Publikation aus dem Jahr 2008 eine, unter anderem auf dem Vorliegen von NAWM basierende, schwache Korrelation zwischen MRT-gestützter Messbarkeit des entzündlichen MS-Geschehens und dem Auftreten klinischer Folgeschäden; auch speziell bei pädiatrischen Patienten (Banwell und Sled 2008).

Die Zusammenschau dieser drei zitierten Quellen unter Berücksichtigung dieser Studie lässt nicht nur den bereits formulierten Schluss zu, dass eine optimierte OCT die unter anderem durch die NAWM verursachte diagnostische Lücke zu schließen helfen kann, sondern unterstreicht auch die Chance einer treffenden Diagnosestellung besonders bei frühzeitiger Anwendung der OCT zur Begleitung von neu aufgetretenen MStypischen Symptomen.

Ebenfalls von großem Interesse sind die etablierten Diagnosekriterien. Das namhafte Kategorisierungssystem nach McDonald et al. aus dem Jahr 2001 mit dem Titel „Recommended Diagnostic Criteria for Multiple Sclerosis: Guidelines from the International Panel on the Diagnosis of Multiple Sclerosis" (McDonald et al. 2001) kann in dieser Studie nur teilweise Anwendung finden, da sich die Diagnosekriterien nach McDonald eher auf mit dem Kontrastmittel Gadolinium angereicherte MS-typische Läsionsherde zum Zweck der Erstdiagnose beziehen. In dieser Studie wurden allerdings keine Kontrastmittel-gestützten Sequenzen ausgewertet, sondern native T2gewichtete Aufnahmen bei Patienten mit diagnostizierter MS. Die McDonald-Kriterien erklären das Vorliegen von 9 in der T2-Wichtung hyperintens erscheinenden Läsionen gegenüber dem Vorliegen einer Gadolinium anreichernden Läsion für gleichwertig. Da in dieser Studie drei Studienteilnehmer insgesamt jeweils weniger als 9 Läsionen aufweisen und daher trotz manifester MS ausgeschlossen werden müssten, kommt für die präzisere Einteilung eher das Klassifikationssystem nach Paty et al. mit dem Titel „MRI in the diagnosis of MS: a prospective study with comparison of clinical evaluation, evoked potentials, oligoclonal banding, and CT" aus dem Jahr 1988 in Frage (Paty et al. 1988). Die Datenauswertung der 45 Teilnehmer dieser Studie ergab für alle Patienten das Vorliegen von mindestens 5 Einzelläsionen und damit eine weitgehende Vereinbarkeit mit den Kriterien der Publikation von 1988, welche das Vorliegen von 4 Einzelläsionen voraussetzen. 
Es stellt sich die Frage, ob für pädiatrische Patienten gegebenenfalls ein eigener Kriterienkatalog angelegt werden sollte. Hahn et al. haben dies in Ihrer Publikation unter dem Gesichtspunkt der Schubabhängigkeit untersucht (Hahn et al. 2004). Hier wurden jeweils Summe und Volumen der MS-typischen Läsionsherde in einer 20 pädiatrische Patienten umfassenden Kohorte bestimmt. Hahn et al. beobachteten eine eingeschränkte Anwendbarkeit der für die adulte MS konzipierten Diagnosekriterien nach McDonald. Die in dieser Studie erhobenen Parameter, wie beispielsweise jene zur Topographie, sowie die im vorangegangenen Ergebnisteil ausführlich präsentierten Datensätze zu Anzahl und Volumen der Läsionen, könnten nebst anderen Datensätzen, welche sich beispielsweise mit der Läsionsmorphologie befassen, bei der Erstellung eines Klassifikationssystems für MS des Kindes- und Jugendalters unterstützende Verwendung finden.

\section{Ausblick}

Im Licht der vorangegangenen Ergebnisdiskussion und der ursprünglichen Fragestellung dieser Arbeit lässt sich abschließend festhalten, dass sich klare Hinweise darauf ergeben, dass die Aussagekraft der OCT bei pädiatrischer MS mit der von MRT und EDSS vergleichbar ist. Die Aufnahme der OCT als fester Bestandteil von Diagnoseund Verlaufsbeobachtungsprotokollen der Multiplen Sklerose bei pädiatrischen Patienten ohne bilaterale NNO-Anamnese, ergänzend zur Nutzung von diagnostischen Instrumenten wie dem MRT und der EDSS-gestützten Erfassung der Klinik, ist somit als nützlich einzustufen. 


\section{$5 \quad$ Zusammenfassung}

Retrospektive Evaluation retinaler Nervenfaserschichtdicke mit der cerebralen T2wLäsionslast im MRT sowie dem Expanded Disability Status Scale (EDSS) bei pädiatrischen Patienten mit Multipler Sklerose

Im Rahmen einer retrospektiv vergleichenden Studie zur Multiplen Sklerose (MS) bei pädiatrischen Patienten wurde eine Evaluation bestehender Datensätze hinsichtlich der Vergleichbarkeit der Aussagekraft von Magnetresonanztomographie (MRT) und klinischen Parametern wie dem EDSS mit der Optischen Kohärenztomographie (OCT) in Bezug auf Nervenfaseratrophie bei an MS erkrankten pädiatrischen Patienten vorgenommen.

Material und Methoden: Bei 45 pädiatrischen Patienten im Alter zwischen 9,75 und 19 Jahren (31 weiblich) wurden Anzahl und Volumen supratentorieller und zerebellärer Läsionen im T2-gewichteten MRT softwaregestützt bestimmt (MIPAV, NIH, Bethesda, MD, USA; FSL, Version 5.0.7, Analysis Group, FMRIB, Oxford, GB) und mit einer etwaigen Abnahme der durch die OCT erhobenen Retinal Nerve Fiber Layer Thickness (RNFLT) verglichen. Zur Erstellung der MRT-Sequenzen wurde ein von SIEMENS hergestellter Magnetresonanztomograph der Baureihe MAGNETOM mit einer Feldstärke von 3 Tesla verwendet. Die OCT-Aufnahmen wurden mithilfe eines von CARL ZEISS MEDITEC hergestellten Optischen Kohärenztomographen der Baureihe CIRRUS HD-OCT (4000-3300) erstellt. Die klinischen Daten und Parameter stammen aus Aufzeichnungen der Kinderklinik der UMG.

Ergebnisse: Die Läsionslast korrelierte invers signifikant mit der retinalen Nervenfaserschichtdicke. Hinsichtlich klinischer Parameter konnte aufgezeigt werden, dass eine Abnahme der RNFLT signifikant mit einer Zunahme des EDSS (hier allerdings bei nicht-adjustiertem $\mathrm{p}$-Wert), der Schubsumme und der Krankheitsdauer korrelierte. Ein signifikanter Zusammenhang mit dem Patientenalter bestand nicht.

Diskussion: Die Ergebnisse sprechen für die Durchführung einer RNFLT-Bestimmung als Begleitung für die etablierten MRT-Untersuchungen und EDSS-Erhebungen bei Diagnostik und Verlaufskontrolle der pädiatrischen MS. 


\section{Publikation}

Vorläufige Ergebnisse dieser Studie wurden auf der gemeinsamen Tagung der Bielschowsky-Gesellschaft für Schielforschung und Neuroophthalmologie sowie des Berufsverbandes der Orthoptistinnen Deutschlands, welche vom 21.11.2015 bis zum 22.11.2015 in Mainz stattfand, am zweiten Tagungstag präsentiert. 


\section{Literaturverzeichnis}

Abalo-Lojo JM, Limeres CC, Gómez MA, Baleato-González S, Cadarso-Suárez C, Capeáns-Tomé C, Gonzalez F (2014): Retinal nerve fiber layer thickness, brain atrophy, and disability in multiple sclerosis patients. J Neuro-Ophthalmol $\underline{34}$, 23-28

Absinta M, Rocca MA, Moiola L, Copetti M, Milani N, Falini A, Comi G, Filippi M (2011): Cortical lesions in children with multiple sclerosis. Neurology $\underline{76}, 910-913$

Adams CW, Abdulla YH, Torres EM, Poston RN (1987): Periventricular lesions in multiple sclerosis: their perivenous origin and relationship to granular ependymitis. Neuropathol Appl Neurobiol 13, 141-152

Alasil T, Wang K, Keane PA, Lee H, Baniasadi N, de Boer JF, Chen TC (2013): Analysis of normal retinal nerve fiber layer thickness by age, sex, and race using spectral domain optical coherence tomography. J Glaucoma 22, 532-541

Ansar Ahmed S, Penhale WJ, Talal N (1985): Sex hormones, immune responses, and autoimmune diseases. Mechanisms of sex hormone action. Am J Pathol $\underline{121}$, 531-551

Atlas Multiple Sclerosis Resources In The World 2008, World Health Organization, Multiple Sclerosis International Federation

Altas of MS 2013, Multiple Sclerosis International Federation

Banwell BL, Anderson PE (2005): The cognitive burden of multiple sclerosis in children. Neurology $\underline{64}, 891-894$

Banwell BL, Sled JG (2008): Starting early: MRI evidence of gray matter atrophy in children with multiple sclerosis. Neurology $\underline{70}, 1065-1066$

Barkhof $F$ (2002): The clinico-radiological paradox in multiple sclerosis revisited. Curr Opin Neurol 15, 239-245

Bock M, Brandt AU, Dörr J, Kraft H, Weinges-Evers N, Gaede G, Pfueller CF, Herges K, Radbruch H, Ohlraun S, et al. (2010): Patterns of retinal nerve fiber layer loss in multiple sclerosis patients with or without optic neuritis and glaucoma patients. Clin Neurol Neurosurg 112, 647-652

Bodini B, Chard D, Altmann DR, Tozer D, Miller DH, Thompson AJ, Wheeler-Kingshott C, Ciccarelli $O$ (2016): White and gray matter damage in primary progressive MS: The chicken or the egg? Neurology $\underline{86}, 170-176$

Boiko A, Vorobeychik G, Paty D, Devonshire V, Sadovnick D, University of British Columbia MS Clinic Neurologists (2002): Early onset multiple sclerosis: a longitudinal study. Neurology $\underline{59}, 1006-1010$ 
Calabrese M, Filippi M, Rovaris M, Mattisi I, Bernardi V, Atzori M, Favaretto A, Barachino L, Rinaldi L, Romualdi C, et al. (2008): Morphology and evolution of cortical lesions in multiple sclerosis. A longitudinal MRI study. Neurolmage $\underline{42}$, $1324-1328$

Calabrese M, Filippi M, Gallo P (2010): Cortical lesions in multiple sclerosis. Nat Rev Neurol $\underline{6}, 438-444$

Chabas D, Castillo-Trivino T, Mowry EM, Strober JB, Glenn OA, Waubant E (2008): Vanishing MS T2-bright lesions before puberty: a distinct MRI phenotype? Neurology 71, 1090-1093

Chitnis T, Glanz B, Jaffin S, Healy B (2009): Demographics of pediatric-onset multiple sclerosis in an MS center population from the Northeastern United States. Mult Scler $\underline{15}, 627-631$

Compston A (1999): The genetic epidemiology of multiple sclerosis. Philos Trans R Soc Lond B Biol Sci 354, 1623-1634

Confavreux C, Vukusic S (2006): Age at disability milestones in multiple sclerosis. Brain J Neurol $\underline{129}$, 595-605

Davis FA (2014): The clinico-radiological paradox in multiple sclerosis: novel implications of lesion size. Mult Scler $\underline{20}, 515-516$

Dörr J, Wernecke KD, Bock M, Gaede G, Wuerfel JT, Pfueller CF, Bellmann-Strobl J, Freing A, Brandt AU, Friedemann P (2011): Association of retinal and macular damage with brain atrophy in multiple sclerosis. PloS One $\underline{6}$, e18132

Duquette P, Murray TJ, Pleines J, Ebers GC, Sadovnick D, Weldon P, Warren S, Paty DW, Upton A, Hader W (1987): Multiple sclerosis in childhood: clinical profile in 125 patients. J Pediatr 111, 359-363

Ebers GC, Koopman WJ, Hader W, Sadovnick AD, Kremenchutzky M, Mandalfino P, Wingerchuk DM, Baskerville J, Rice GP (2000): The natural history of multiple sclerosis: a geographically based study: 8: familial multiple sclerosis. Brain J Neurol 123 Pt 3, 641-649

Epplen C, Jäckel S, Santos EJ, D'Souza M, Poehlau D, Dotzauer B, Sindern E, Haupts M, Rüde KP, Weber F, et al. (1997): Genetic predisposition to multiple sclerosis as revealed by immunoprinting. Ann Neurol $\underline{41}, 341-352$

Filippi M, Bozzali M, Rovaris M, Gonen O, Kesavadas C, Ghezzi A, Martinelli V, Grossman RI, Scotti G, Comi G, Falini A (2003): Evidence for widespread axonal damage at the earliest clinical stage of multiple sclerosis. Brain J Neurol $\underline{126}$, 433-437

Filippi M, Rocca MA, Ciccarelli O, De Stefano N, Evangelou N, Kappos L, Rovira A, Sastre-Garriga J, Tintorè M, Frederiksen JL, et al. (2016): MRI criteria for the 
diagnosis of multiple sclerosis: MAGNIMS consensus guidelines. Lancet Neurol $\underline{15}, 292-303$

Ge Y (2006): Seeing is believing: in vivo evolution of multiple sclerosis pathology with magnetic resonance. Top Magn Reson Imaging TMRI 17, 295-306

Ghezzi A, Pozzilli C, Liguori M, Marrosu MG, Milani N, Milanese C, Simone I, Zaffaroni M (2002): Prospective study of multiple sclerosis with early onset. Mult Scler $\underline{8}$, 115-118

Hahn CD, Shroff MM, Blaser SI, Banwell BL (2004): MRI criteria for multiple sclerosis: Evaluation in a pediatric cohort. Neurology $\underline{62}, 806-808$

Haliloglu G, Anlar B, Aysun S, Topcu M, Topaloglu H, Turanli G, Yalnizoglu D (2002): Gender prevalence in childhood multiple sclerosis and myasthenia gravis. J Child Neurol 17, 390-392

Hanefeld F (1992): Multiple sclerosis in childhood. Curr Opin Neurol Neurosurg $\underline{5}$, 359363

Hanefeld F, Bauer HJ, Christen HJ, Kruse B, Bruhn H, Frahm J (1991): Multiple sclerosis in childhood: report of 15 cases. Brain Dev $\underline{13}, 410-416$

Hummel H: Multiple Sklerose im Kindes- und Jugendalter - klinische und neuroradiologische Besonderheiten. Med. Diss. Göttingen 2015

Jacobson DL, Gange SJ, Rose NR, Graham NM (1997): Epidemiology and estimated population burden of selected autoimmune diseases in the United States. Clin Immunol Immunopathol 84, 223-243

Kallenbach K, Frederiksen J (2007): Optical coherence tomography in optic neuritis and multiple sclerosis: a review. Eur J Neurol 14, 841-849

Kidd D, Barkhof F, McConnell R, Algra PR, Allen IV, Revesz T (1999): Cortical lesions in multiple sclerosis. Brain J Neurol 122 (Pt 1), 17-26

Kuchling J, Ramien C, Bozin I, Dörr J, Harms L, Rosche B, Niendorf T, Paul F, Sinnecker T, Wuerfel J (2014): Identical lesion morphology in primary progressive and relapsing-remitting MS--an ultrahigh field MRI study. Mult Scler 20, 1866-1871

Kumar DR, Aslinia F, Yale SH, Mazza JJ (2011): Jean-Martin Charcot: The Father of Neurology. Clin Med Res $\underline{9}$, 46-49

Kurtzke JF (1983): Rating neurologic impairment in multiple sclerosis: an expanded disability status scale (EDSS). Neurology $\underline{33}$, 1444-1452

Kutzelnigg A, Lassmann H (2005): Cortical lesions and brain atrophy in MS. J Neurol Sci $\underline{233}, 55-59$

Lamirel C, Newman NJ, Biousse V (2010): Optical Coherence Tomography (OCT) in Optic Neuritis and Multiple Sclerosis. Rev Neurol (Paris) 166, 978-986 
Levin LI, Munger KL, O'Reilly EJ, Falk KI, Ascherio A (2010): Primary infection with the Epstein-Barr virus and risk of multiple sclerosis. Ann Neurol 67, 824-830

Lin R, Charlesworth J, van der Mei I, Taylor BV (2012): The genetics of multiple sclerosis. Pract Neurol 12, 279-288

Llufriu S, Pujol T, Blanco Y, Hankiewicz K, Squarcia M, Berenguer J, Villoslada P, Graus F, Saiz A (2010): T2 hypointense rims and ring-enhancing lesions in MS. Mult Scler $\underline{16}, 1317-1325$

Lublin F (2005): History of modern multiple sclerosis therapy. J Neurol 252 Suppl 3 , iii3iii9

Marcus DS, Olsen TR, Ramaratnam M, Buckner RL (2007): The Extensible Neuroimaging Archive Toolkit: an informatics platform for managing, exploring, and sharing neuroimaging data. Neuroinformatics $\underline{5}, 11-34$

Martinez-Lapiscina EH, Arnow S, Wilson JA, Saidha S, Preiningerova JL, Oberwahrenbrock T, Brandt AU, Pablo LE, Guerrieri S, Gonzalez I, et al. (2016): Retinal thickness measured with optical coherence tomography and risk of disability worsening in multiple sclerosis: a cohort study. Lancet Neurol $\underline{15}$, 574-584

McDonald WI, Compston A, Edan G, Goodkin D, Hartung HP, Lublin FD, McFarland HF, Paty DW, Polman CH, Reingold SC, et al. (2001): Recommended diagnostic criteria for multiple sclerosis: guidelines from the International Panel on the diagnosis of multiple sclerosis. Ann Neurol $\underline{50}, 121-127$

Murray TJ (2009): The history of multiple sclerosis: the changing frame of the disease over the centuries. J Neurol Sci 277 Suppl 1, S3-8

Oberwahrenbrock T, Weinhold M, Mikolajczak J, Zimmermann H, Paul F, Beckers I, Brandt AU (2015): Reliability of Intra-Retinal Layer Thickness Estimates. PloS One 10 , e0137316

O'Connor P, Canadian Multiple Sclerosis Working Group (2002): Key issues in the diagnosis and treatment of multiple sclerosis. An overview. Neurology $\underline{59}$, S133

Orton S-M, Herrera BM, Yee IM, Valdar W, Ramagopalan SV, Sadovnick AD, Ebers GC, Canadian Collaborative Study Group (2006): Sex ratio of multiple sclerosis in Canada: a longitudinal study. Lancet Neurol 5 , 932-936

Ozakbas S, Idiman E, Baklan B, Yulug B (2003): Childhood and juvenile onset multiple sclerosis: clinical and paraclinical features. Brain Dev $\underline{25}, 233-236$

Parisi V, Manni G, Spadaro M, Colacino G, Restuccia R, Marchi S, Bucci MG, Pierelli F (1999): Correlation between morphological and functional retinal impairment in multiple sclerosis patients. Invest Ophthalmol Vis Sci 40, 2520-2527 
Patel Y, Bhise V, Krupp L (2009): Pediatric multiple sclerosis. Ann Indian Acad Neurol $\underline{12}, 238-245$

Paty DW, Oger JJ, Kastrukoff LF, Hashimoto SA, Hooge JP, Eisen AA, Eisen KA, Purves SJ, Low MD, Brandejs V (1988): MRI in the diagnosis of MS: a prospective study with comparison of clinical evaluation, evoked potentials, oligoclonal banding, and CT. Neurology 38, 180-185

Pohl D: Epidemiologie und Genetik. In Zettl U K, Köhler W, Buchmann J (Hrsg.): Multiple Sklerose im Kinder- und Jugendalter. Thieme, Stuttgart 2007, 13-14

Polman CH, Reingold SC, Banwell B, Clanet M, Cohen JA, Filippi M, Fujihara K, Havrdova E, Hutchinson M, Kappos L, et al. (2011): Diagnostic criteria for multiple sclerosis: 2010 revisions to the McDonald criteria. Ann Neurol 69, 292-302

Pugliatti M, Rosati G, Carton H, Riise T, Drulovic J, Vécsei L, Milanov I (2006): The epidemiology of multiple sclerosis in Europe. Eur J Neurol $\underline{13}, 700-722$

Ramagopalan SV, Valdar W, Criscuoli M, DeLuca GC, Dyment DA, Orton S-M, Yee IM, Ebers GC, Sadovnick AD, Canadian Collaborative Study Group (2009): Age of puberty and the risk of multiple sclerosis: a population based study. Eur J Neurol $\underline{16}, 342-347$

Reinhardt K, Weiss S, Rosenbauer J, Gärtner J, von Kries R (2014): Multiple sclerosis in children and adolescents: incidence and clinical picture - new insights from the nationwide German surveillance (2009-2011). Eur J Neurol 21, 654-659

Renoux C, Vukusic S, Mikaeloff Y, Edan G, Clanet M, Dubois B, Debouverie M, Brochet B, Lebrun-Frenay C, Pelletier J, et al. (2007): Natural history of multiple sclerosis with childhood onset. N Engl J Med 356, 2603-2613

Ruggieri M, Polizzi A, Pavone L, Grimaldi LM (1999): Multiple sclerosis in children under 6 years of age. Neurology $\underline{53}, 478-484$

Sadovnick AD, Dyment D, Ebers GC (1997): Genetic epidemiology of multiple sclerosis. Epidemiol Rev $\underline{19}, 99-106$

Salchow DJ, Oleynikov YS, Chiang MF, Kennedy-Salchow SE, Langton K, Tsai JC, AlAswad LA (2006): Retinal nerve fiber layer thickness in normal children measured with optical coherence tomography. Ophthalmology $\underline{113}$, 786-791

Scheel M, Finke C, Oberwahrenbrock T, Freing A, Pech L-M, Schlichting J, Sömmer C, Wuerfel J, Paul F, Brandt AU (2014): Retinal nerve fibre layer thickness correlates with brain white matter damage in multiple sclerosis: a combined optical coherence tomography and diffusion tensor imaging study. Mult Scler Houndmills Basingstoke Engl 20, 1904-1907

Schneider E, Zimmermann H, Oberwahrenbrock T, Kaufhold F, Kadas EM, Petzold A, Bilger F, Borisow N, Jarius S, Wildemann B, et al. (2013): Optical Coherence Tomography Reveals Distinct Patterns of Retinal Damage in Neuromyelitis Optica and Multiple Sclerosis. PloS One $\underline{8}$, e66151 
Shaw CM, Alvord EC (1987): Multiple sclerosis beginning in infancy. J Child Neurol 2 , 252-256

Sinnecker T, Dörr J, Pfueller CF, Harms L, Ruprecht K, Jarius S, Brück W, Niendorf T, Wuerfel J, Paul F (2012): Distinct lesion morphology at 7-T MRI differentiates neuromyelitis optica from multiple sclerosis. Neurology $\underline{79}, 708-714$

Sinnecker T, Oberwahrenbrock T, Metz I, Zimmermann H, Pfueller CF, Harms L, Ruprecht K, Ramien C, Hahn K, Brück W, et al. (2015): Optic radiation damage in multiple sclerosis is associated with visual dysfunction and retinal thinning--an ultrahigh-field MR pilot study. Eur Radiol 25, 122-131

Trip SA, Schlottmann PG, Jones SJ, Altmann DR, Garway-Heath DF, Thompson AJ, Plant GT, Miller DH (2005): Retinal nerve fiber layer axonal loss and visual dysfunction in optic neuritis. Ann Neurol $\underline{58}, 383-391$

Waubant E, Chabas D, Okuda DT, Glenn O, Mowry E, Henry RG, Strober JB, Soares B, Wintermark M, Pelletier D (2009): Difference in disease burden and activity in pediatric patients on brain magnetic resonance imaging at time of multiple sclerosis onset vs adults. Arch Neurol $\underline{66}$, 967-971

Whitacre CC (2001): Sex differences in autoimmune disease. Nat Immunol $\underline{2}, 777-780$

Yeh EA, Marrie RA, Reginald YA, Buncic JR, Noguera AE, O'Mahony J, Mah JK, Banwell B, Costello F, Canadian Pediatric Demyelinating Disease Network (2014): Functional-structural correlations in the afferent visual pathway in pediatric demyelination. Neurology $\underline{83}, 2147-2152$

\section{Internetquellen:}

http://www.zeiss.de/meditec/produkte/ophthalmologie/retina/diagnose/optischekohaerenztomographie/cirrus-familie/cirrus-hd-oct.html; Zugriff am 10.12.2016, 12:29Uhr

http://www.fonar.com/glossary.htm; Zugriff am 10.12.2016, 12:27Uhr 


\section{Lebenslauf}

Am 28.02.1990 wurde ich als zweites von drei Kindern von Saja Alwa und Ibrahim AlBourini in Hannover geboren. Dort absolvierte von 1996 bis 2009 meine Schulbildung. 2009 wurde mir an der Elsa-Brändström-Schule das Abitur mit der Note 1,3 verliehen. Anschließend leistete ich bis zum Frühjahr 2010 meinen Zivildienst beim Kommunalen Seniorenservice der Stadt Hannover ab. Darauf folgten ein zweimonatiges Krankenpflegepraktikum am International Neuroscience Institute sowie eine Gasthörerschaft an der Leibniz-Universität Hannover. Am daran angeschlossen Institut für Entwicklungsforschung und Strukturplanung war ich von Januar bis April 2011 beschäftigt. Mit Beginn des Sommersemesters 2011 trat ich das Studium der Humanmedizin an der Georg-August-Universität Göttingen an; dieses beendete ich im Sommersemester am 16.05.2017 mit der Gesamtnote Gut. Einen Teil des Praktischen Jahres verbrachte ich als PROMOS-Stipendiat des Deutschen Akademischen Austauschdienstes (DAAD) am College of Medicine der Seoul National University in Südkorea. Des Weiteren war ich während des Studiums mehrfach als studentische Hilfskraft tätig: Im vierten Semester (Wintersemester 2012/2013) am Zentrum für Anatomie sowie von 2015 bis 2017 am Institut für Diagnostische und Interventionelle Radiologie. Seit Juli 2017 bin ich im selben Institut der Universitätsmedizin Göttingen als Assistenzarzt tätig. 